Revue des patrimoines

$31 \mid 2017$

Patrimoines de la santé : essais de définition - enjeux de conservation

\title{
Le cimetière intercommunal de la Fontaine Saint- Martin à Valenton, une réalisation oubliée de Robert Auzelle
}

The cemetery of "La Fontaine Saint-Martin " in Valenton, a little known realization by Robert Auzelle

Isabelle Duhau

\section{OpenEdition}

Journals

Édition électronique

URL : http://journals.openedition.org/insitu/14255

DOI : 10.4000/insitu.14255

ISSN : 1630-7305

Éditeur

Ministère de la Culture

Référence électronique

Isabelle Duhau, « Le cimetière intercommunal de la Fontaine Saint-Martin à Valenton, une réalisation oubliée de Robert Auzelle », In Situ [En ligne], 31 | 2017, mis en ligne le 21 février 2017, consulté le 09 octobre 2020. URL : http://journals.openedition.org/insitu/14255; DOI : https://doi.org/10.4000/ insitu. 14255

Ce document a été généré automatiquement le 9 octobre 2020.

\section{c) (1) $९$}

In Situ Revues des patrimoines est mis à disposition selon les termes de la licence Creative Commons Attribution - Pas d'Utilisation Commerciale - Pas de Modification 4.0 International. 


\title{
Le cimetière intercommunal de la Fontaine Saint-Martin à Valenton, une réalisation oubliée de Robert Auzelle
}

\author{
The cemetery of " La Fontaine Saint-Martin » in Valenton, a little known \\ realization by Robert Auzelle
}

Isabelle Duhau

L'architecte français Robert Auzelle ${ }^{1}$ (1913-1983) est reconnu et étudié pour ses écrits et son enseignement en matière d'urbanisme, recherches qu'il mit en œuvre à partir des années 1950 dans le quartier de la Plaine à Clamart (Hauts-de-Seine). Il est également réputé comme spécialiste de l'architecture funéraire grâce à d'autres textes ${ }^{2}$ et à la réalisation, à partir de 1946, du premier véritable cimetière paysager français du $\mathrm{xx}^{\mathrm{e}}$ siècle, toujours à Clamart. Ce premier cimetière intercommunal d'île-de-France inaugure une série de nécropoles inédites par leur ampleur. Cependant, les autres ensembles conçus par l'architecte comme ses nombreux projets non réalisés demeurent assez peu connus, y compris le cimetière intercommunal des Joncherolles à Pierrefitte et Villetaneuse (Seine-Saint-Denis), projeté à partir de 1958. Chargée jusqu'en 2010 du département du Val-de-Marne (Val-de-Marne) au service de l'Inventaire général du patrimoine culturel de la région Île-de-France, nous avons eu l'occasion d'étudier le troisième cimetière intercommunal que l'architecte réalisa, cette fois à Valenton ${ }^{3}$.

Dans cette ville du sud-est parisien, Auzelle déploie, une fois encore, toutes ses idées pour réinventer le cimetière français, cimetière dans lequel il voudrait faire régner un esprit des lieux retrouvé, une harmonie propice au recueillement et à une forme de sacralité restaurée; ce "cimetière paysager» proposerait la synthèse entre le «cimetière parc» américain, celui «forestier», de tradition germanique, et le "cimetière architectural» méditerranéen ${ }^{4}$. Récusant «la monotone découpe orthogonale des nécropoles suburbaines [françaises qui] a dépoétisé les champs des morts» et "l'entassement désolant» des sections de concessions au quadrillage 
compact, l'architecte dresse le constat que «le plus souvent c'est à l'étranger qu'il faudra chercher des exemples de solutions séduisantes, aussi bien pour une conception plus souple et plus aérée de la composition que pour une esthétique plus sûre et plus modeste du monument individuel $»^{5}$. En effet, Auzelle œuvre également durant toute sa carrière à la promotion d'une approche renouvelée des sépultures elles-mêmes, perpétuellement surpris "devant l'abondance de l'effarante production funéraire hétéroclite des derniers siècles » dans laquelle on pourrait selon lui établir un « musée des horreurs $»^{6}$. Cette piètre production révèle le triomphe des manifestations individualistes au détriment de l'âme des cimetières contemporains, ces derniers ayant perdu, à ses yeux, le caractère immémorial et intemporel des cimetières anciens.

3 Le projet de Valenton succède à un premier projet prévu à Créteil, pour le compte des mêmes communes. Sa réalisation, au début des années 1970, se conforme dans l'ensemble aux dessins de l'architecte. Les interventions artistiques y occupent une place importante, Auzelle intégrant des sculptures monumentales commandées à des plasticiens qu'il choisit soigneusement. Cependant, le maitre d'œuvre échoue ici en grande partie dans sa volonté de renouveler l'art funéraire, ne réussissant pas à multiplier les tombes superposées en enfeus et à imposer ses dessins pour l'ensemble des sépultures (fig. 1).

Figure 1

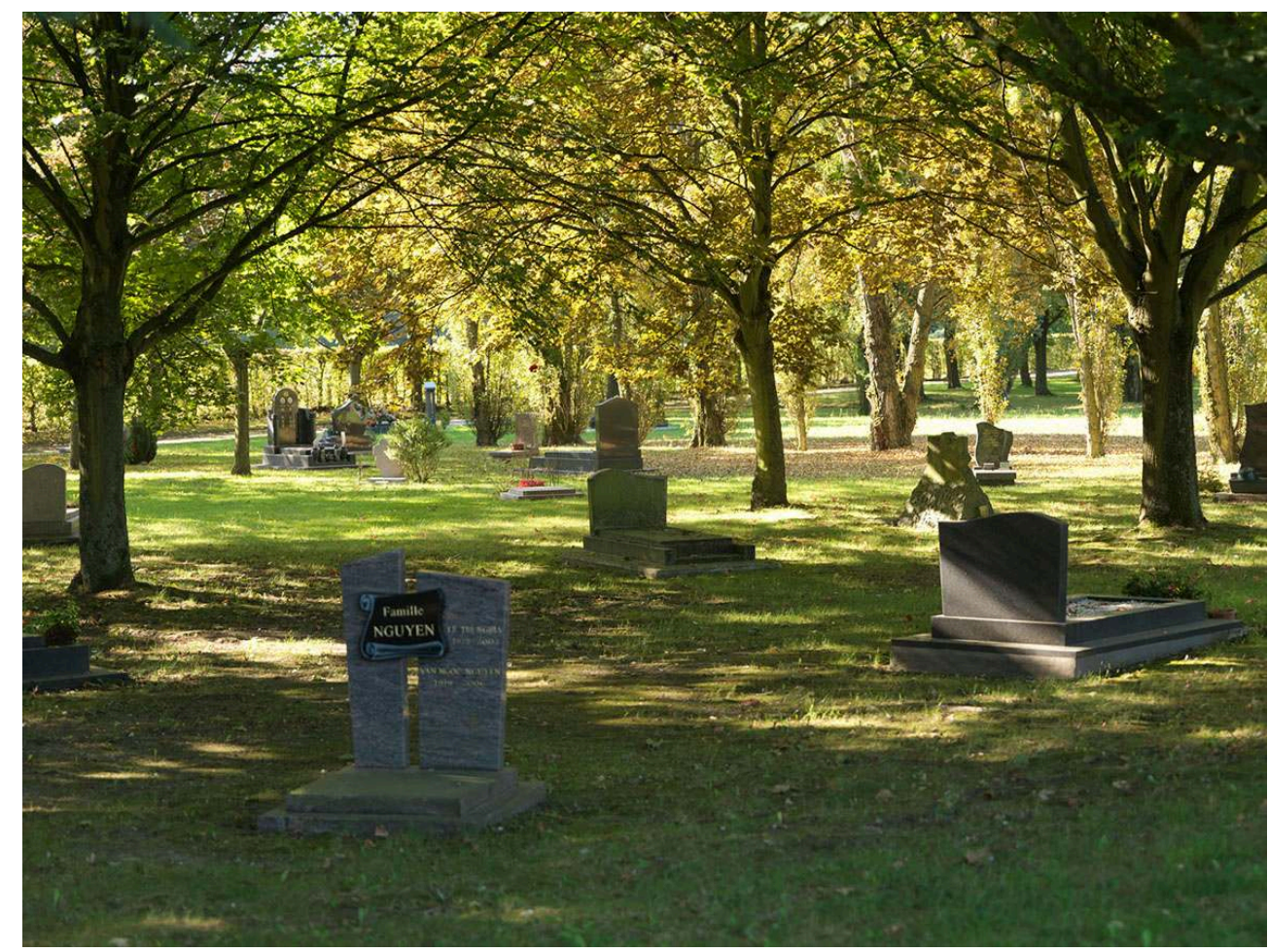

Cimetière de la Fontaine-Saint-Martin, Valenton, zone paysagère accueillant des sépultures isolées. PHOT. ASSELINE, STÉPHANE (20149400010). @ INVENTAIRE gÉNÉRAL DU PATRIMOINE CULTUREL. RÉgION ÎLE-DE-FRANCE, 2014. 


\section{Le cimetière envisagé à Créteil}

4 Au sortir de la Seconde Guerre mondiale, face à la pénurie de places dans leurs nécropoles, les pouvoirs publics et les communes d'île-de-France s'organisent et créent de vastes cimetières intercommunaux, équipements projetés dans le cadre des plans successifs d'aménagement de la région parisienne depuis les années 1920 mais encore jamais mis en œuvre ${ }^{7}$. Dans ce qui est encore le département de la Seine, Bonneuil-surMarne, Charenton-le-Pont, Créteil ${ }^{8}$, Joinville-le-Pont, Maisons-Alfort, Saint-Maur-desFossés et Saint-Maurice (Nogent-sur-Marne ne rejoindra le syndicat qu'après l'ouverture du cimetière et la commune de Valenton n'est pas adhérente) se regroupent pour fonder le « syndicat intercommunal pour la création d'un cimetière à Créteil » dès 1958. Le terrain choisi, environ 30 hectares, est situé au sud de Créteil, sur les coteaux du Mont-Mesly, au-delà de l'ensemble urbain réalisé par Gustave Stoskopf pour la SCIC (Société centrale immobilière de la Caisse des dépôts) à partir de 1955. Le plan d'aménagement intercommunal $\mathrm{n}^{\circ} 9$ de 1961 désigne le site comme une réserve d'espaces verts. Le projet est déclaré d'utilité publique le 5 novembre 1962 et le terrain acquis deux ans plus tard. En 1966, Robert Auzelle est choisi comme architecte de l'opération. Il demande que Pierre Lery ${ }^{9}$ lui soit adjoint, les deux architectes travaillant déjà ensemble sur le projet de cimetière des Joncherolles et sur celui de Villiers-le-Bel $\left(\right.$ Val-d'Oise) ${ }^{10}$ (fig. 2). Auzelle explique "si nous nous sommes déclaré partisan convaincu du cimetière intercommunal, c'est qu'il nous a paru se prêter, mieux que toute autre formule, à cette conception nouvelle qui voudrait traiter le service de la mort comme un service public - au même titre, par exemple, que le service des Eaux ». En effet, l'architecte inclut dans ses réflexions sur le renouvellement du cimetière français son cadre législatif, sa gestion (étude prospective des besoins, typologie de concessions) ou encore ses enjeux économiques (ressources pour son financement et son entretien, conditions d'amortissement) proposant des réformes ainsi que la réalisation de nouveaux dispositifs (ossuaire-nécrologe, crématoire, aire de cérémonie) afin de remédier au diagnostic sévère qu'il dresse ${ }^{11}$. Chacun de ses projets successifs est donc l'occasion de décliner et d'affiner ses recherches théoriques. 


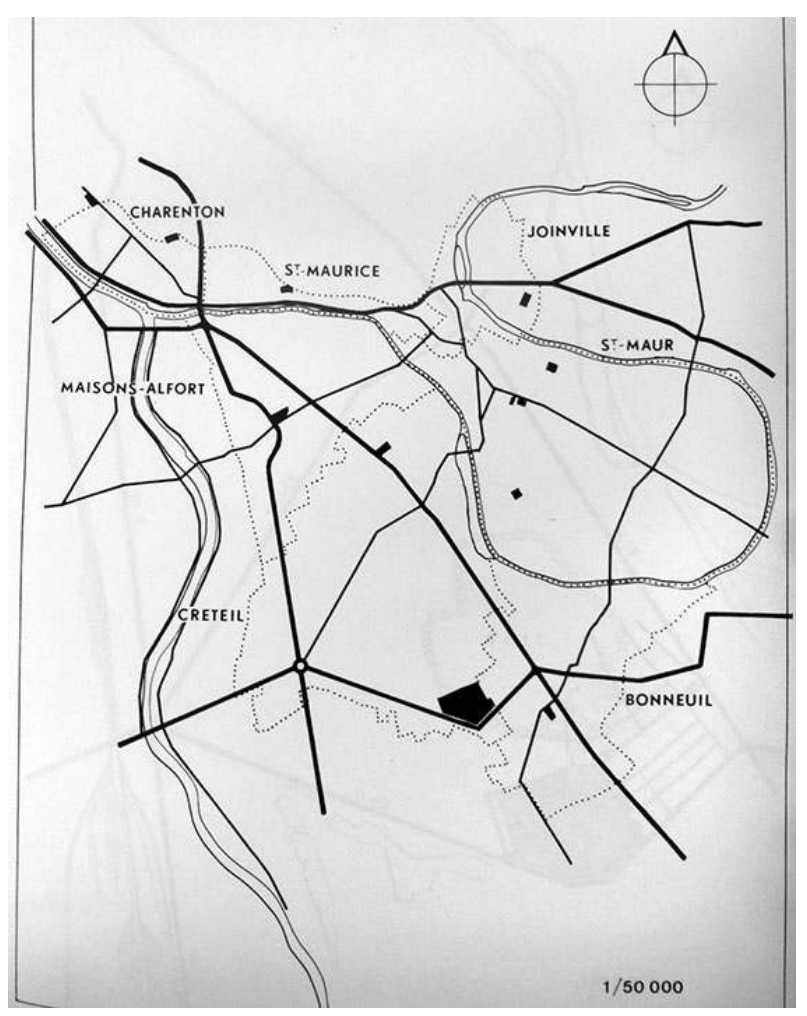

Projet de cimetière du Mont-Mesly, Créteil. PLAN DE Localisation du Terrain, aujourd'hui le long de la route de Pompadour. Dossier d'avant-projet, avril 1966. SIAF/CAPA. Fonds Robert Auzelle 242 IFA. Carton 56, affaire RA66 (cimetière du Mont-Mesly à Créteil).

REPRO. DUHAU, ISABELLE (20149400021). ( ) INVENTAIRE gÉNÉRAL DU PATRIMOINE CULTUREL. RÉgION ÎLE-DE-FRANCE, 2014.

5 Sur la parcelle cristolienne formant une demi-croupe à deux pentes principales très affirmées (dénivelé de 25 à $35 \mathrm{~m}$ sur $500 \mathrm{~m}$ ) suivant des orientations perpendiculaires sud-est/sud-ouest, Auzelle souhaite rationnaliser sa composition afin de réduire les terrassements nécessaires à la création d'aires d'inhumation bien planes et de permettre la réalisation de l'ensemble par tranches successives. Il divise ainsi le terrain en six grandes zones. La première (I), implantée au point haut, à l'angle nord-ouest, accueille "les services", c'est-à-dire les entrées distinctes pour les convois, les visiteurs et les marbriers, les bureaux du syndicat, la conservation, deux logements, les aires de stationnement, les commerces funéraires, les aires de dépôt de matériel, enfin les bâtiments des fossoyeurs. La zone II, disposée en quart de cercle autour de la première, est décomposée en "terrasses hexagonales» étagées sur la pente, la dénivellation entre les terrasses étant utilisée pour placer des tombes superposées sur trois niveaux. Le reste de la pente, au sud-ouest (III), est aménagé en «cimetières rectangulaires " où les quatre niveaux successifs comprennent également des tombes superposées. Sur la seconde partie du versant sud-est, l'architecte place des aires d'inhumation rectangulaires, à la réglementation stricte, s'inscrivant en grands emmarchements dans la pente du terrain, baptisés « les grands degrés » (IV). Au sud, il regroupe les tombes en enfeus (qu'il souhaite nombreux) ainsi que les ossuaires individuels et collectifs ${ }^{12}(\mathrm{~V})$. Enfin, au centre du terrain, à l'intersection des zones II, III, IV et $\mathrm{V}$, et pour mieux les individualiser, il réserve un vaste espace planté, également utilisé pour des inhumations suivant une trame hexagonale d'isolement de 
$22,5 \mathrm{~m}^{2}$, zone baptisée "les tombes sous les arbres"(VI). Les circulations sont nettement décomposées selon deux types, celui des voies à double sens dont le tracé forme un 8 délimitant et desservant les six zones, et celui des circulations secondaires, à sens unique, donnant accès aux aires d'inhumation et pouvant être réalisées en fonction des tranches. Pour la première phase de travaux, Auzelle préconise de prévoir la totalité des circulations à double sens, l'ensemble de la zone de services, un premier bâtiment d'enfeus et un ossuaire.

En additionnant les tombes superposées et celles en enfeus, les sépultures familiales ordinaires (pouvant accueillir quatre corps) et celles de la zone sous les arbres (pour six corps), l'architecte dessine un équipement susceptible de recevoir près de 92000 défunts. Il réussit le tour de force de concevoir un cimetière de grande capacité en évitant la monotonie habituelle de ce type d'équipement grâce à une organisation par zones aux dessins très contrastés et tout en aménageant un espace vert à la composition structurée. Dès son premier avant-projet, il localise les zones engazonnées, celles plantées de fleurs et celles des différentes catégories de plantations. Il détaille les végétaux choisis, bouleaux, chênes, conifères de différentes tailles, zones de buissons, enfin haies taillées. Il réserve ainsi les arbres de plus haute tige (les arbres dominants, représentés par des triangles noirs sur le plan) pour le pourtour du cimetière, le séparant de la ville en plein développement alentour. Il enclot chacune des zones plantées de feuillues par des conifères, arbres au feuillage persistant, afin de mieux les isoler les unes des autres et d'y développer des enclaves de sérénité de plus petite échelle, conformément à son principe de mixité des paysages (fig. 3). 
Figure 3

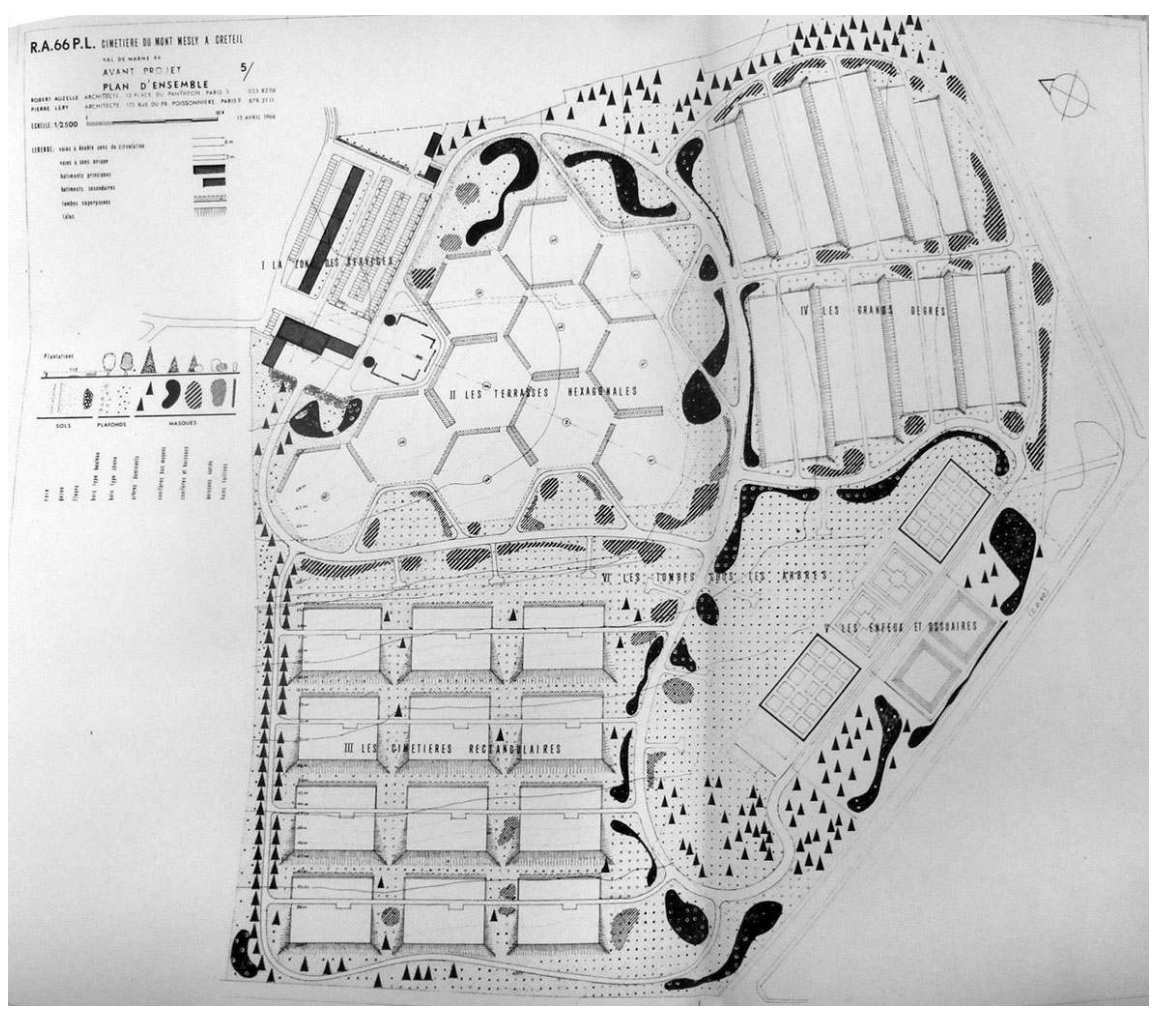

Projet de cimetière du Mont-Mesly, Créteil. Plan d'ensemble. Dossier d'avant-projet, avril 1966. SIAF/ CAPA. Fonds Robert Auzelle 242 IFA. Carton 56, affaire RA66.

REPRO. DUHAU, ISABELLE (20149400022). @ INVENTAIRE gÉNÉRAL DU PATRIMOINE CULTUREL. RÉgION ÎLE-DE-FRANCE, 2014.

7 Auzelle rend son premier avant-projet en avril 1966, esquisse qu'il affine dans les mois qui suivent, allant, fort de sa longue expérience en matière d'aménagements funéraires, jusqu'à faire des suggestions au syndicat afin d'amender le programme. Dans le bâtiment des cérémonies, il ajoute un dépositoire de huit cases, une salle d'autopsie et un bureau de médecin. Il propose également un nouvel élément qui s'impose à ses yeux, $\mathrm{du}$ fait que les habitants résident désormais dans des logements exigus et ne peuvent plus conserver le corps d'un défunt à domicile. Il intègre une salle d'exposition mortuaire, lieu où les familles pourraient garder le corps avant la mise en bière et la cérémonie funèbre, précisant que cet équipement tend à être implanté par les pompes funèbres sous le nom de funérarium (fig. 4). L'architecte répond aux interrogations du syndicat et argumente pour défendre le principe des sépultures en élévation qu'il essaie de promouvoir dans ses différents projets malgré l'hostilité des marbriers et le peu d'enthousiasme des familles, faisant même réaliser des prototypes $^{13}$. 
Figure 4

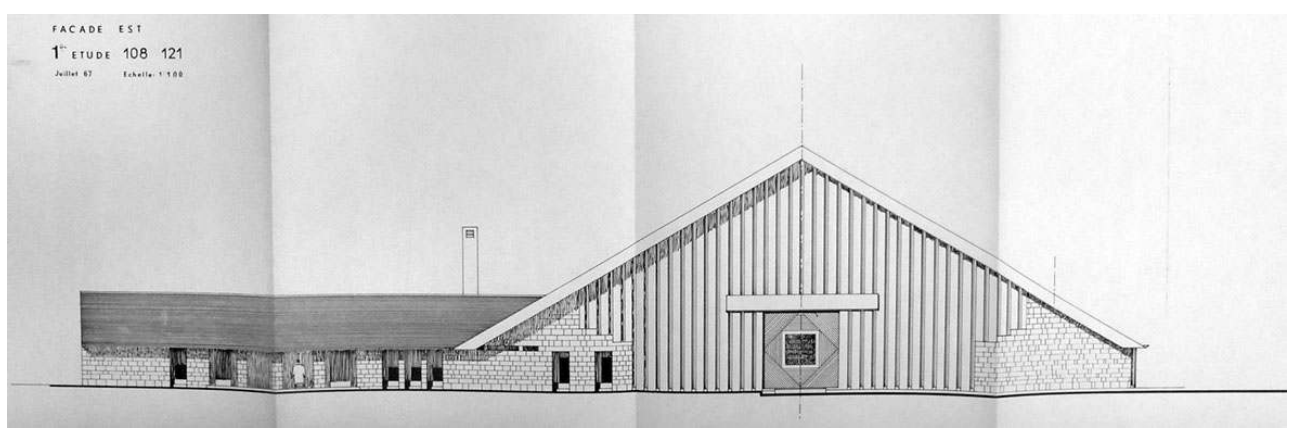

Projet de cimetière du Mont-Mesly, Créteil. Élévation Est des bâtiments de service. À DRoITE L'AIRE

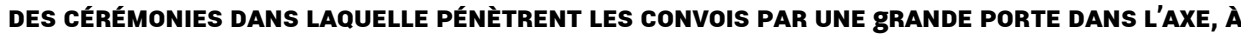
gAUCHE LES BUREAUX DE LA CONSERVATION ET LE LOgEMENT dU CONSERVATEUR. Dossier d'avantprojet, juillet 1967. SIAF/CAPA. Fonds Robert Auzelle 242 IFA. Carton 56, affaire RA66.

REPRO. DUHAU, ISABELLE (20149400023). @ INVENTAIRE gÉNÉRAL DU PATRIMOINE CULTUREL. RÉgION ÎLE-DE-FRANCE, 2014.

Bien que le syndicat redouble ses efforts pour maintenir coûte que coûte la réalisation du futur cimetière, il doit renoncer fin 1967 à l'implanter sur ce premier terrain. Entre temps, les pouvoirs publics et la Ville de Créteil, devenue la préfecture du nouveau département du Val-de-Marne créé en 1964, ont revu leurs projets d'extension urbaine du secteur et entendent poursuivre dans cette zone l'urbanisation du Mont-Mesly.

\section{Le nouveau projet conçu pour le site de Valenton}

9 Malgré la pression foncière, qui atteint les marges de la première couronne parisienne, un second terrain est trouvé, situé au sud du cimetière de Limeil-Brévannes, sur les territoires de Valenton, Limeil et Villeneuve-Saint-Georges. Les 40 hectares de plaine sont bordés au sud-est par l'emprise du futur échangeur A87-A5 (autoroutes qui ne seront finalement pas réalisées) et à l'ouest par le prolongement du CD 94 (aujourd'hui avenue de la Fontaine-Saint-Martin) ${ }^{14}$. À la date de sa conception, il s'agit du plus grand cimetière intercommunal français projeté. En avril 1969, Auzelle, associé cette fois à André Mahé et à Hector Patriotis ${ }^{15}$ pour l'exécution, rend son avant-projet (fig. 5, fig. 6). 
Figure 5

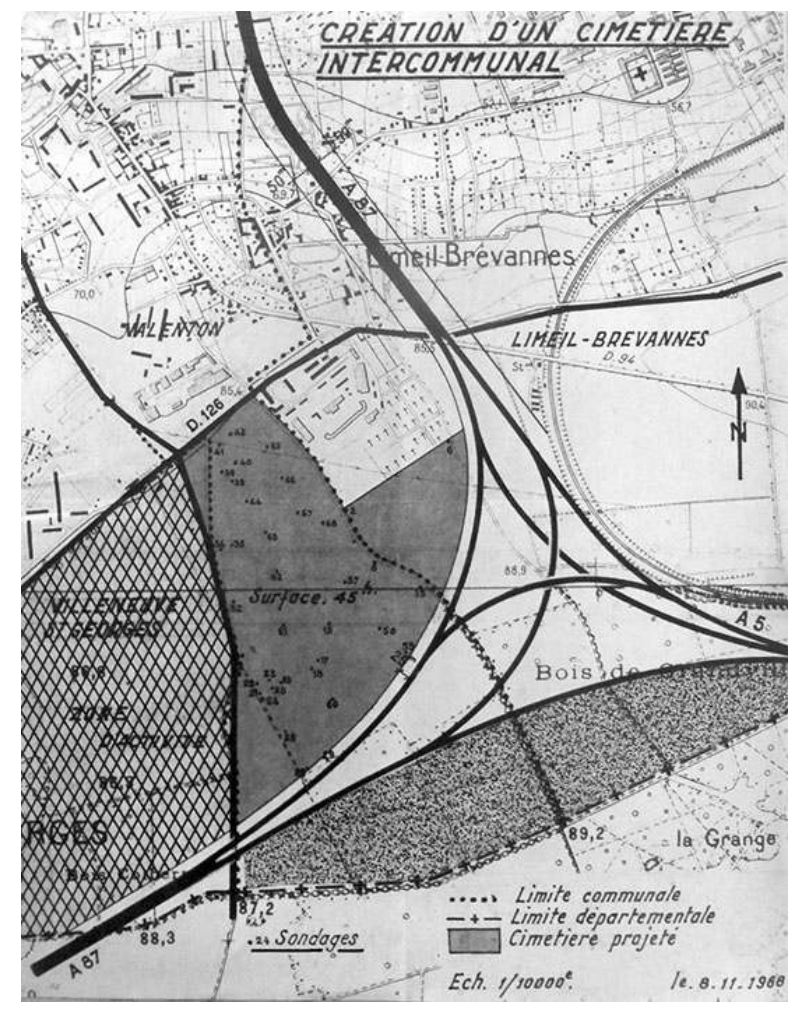

Plan de localisation du terrain finalement retenu, daté de novembre 1968, fourni à Robert Auzelle en même temps que le programme définitif. SIAF/CAPA. Fonds Robert Auzelle 242 IFA. Carton 58, affaire RA82, programme et premières esquisses.

REPRO. DUHAU, ISABELLE (20149400024). @ INVENTAIRE gÉNÉRAL DU PATRIMOINE CULTUREL. RÉgION ÎLE-DE-FRANCE, 2014. 


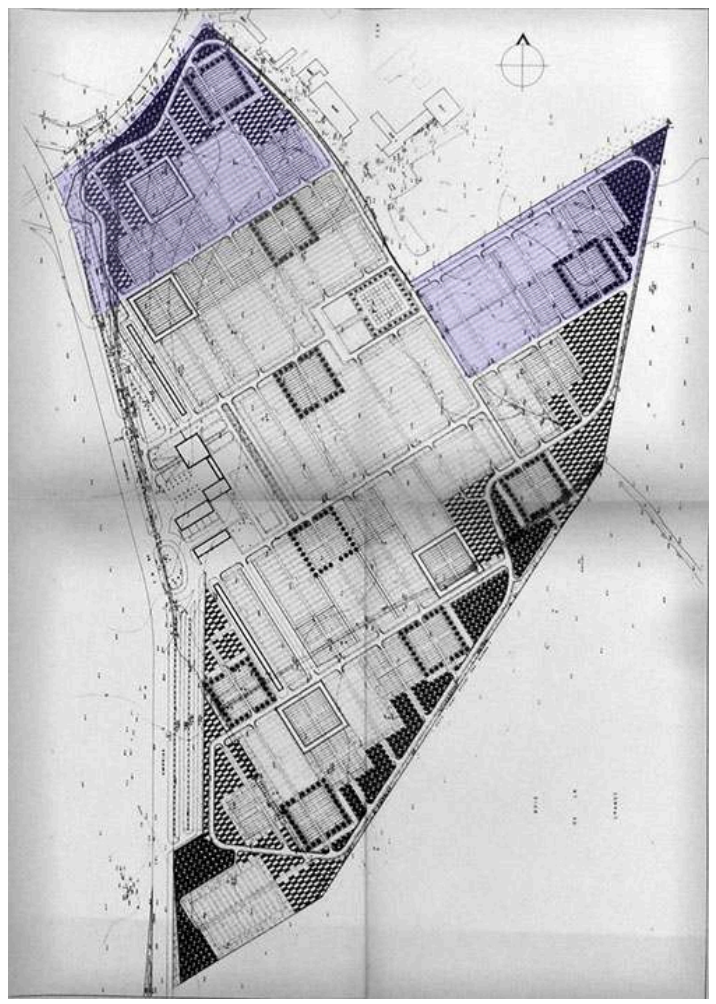

Plan de composition (en mauve les deux zones qui ont été soustraites au cimetière actuel). Dossier d'avant-projet, avril 1969. SIAF/CAPA. Fonds Robert Auzelle 242 IFA. Carton 57, affaire RA82.

REPRO. DUHAU, ISABELLE (20149400029). @ INVENTAIRE gÉNÉRAL DU PATRIMOINE CULTUREL. RÉgION ÎLE-DE-FRANCE, 2014.

Le programme exigeant de placer près de 44000 sépultures, l'architecte n'envisage pas un cimetière paysager trop lâche et la planéité du terrain encourage une composition tramée. En outre, les servitudes des lignes EDF à haute tension qui jouxtent la parcelle interdisent de prescrire des arbres de haute tige dans certaines zones. Fidèle à son sens de la mesure et aux compositions très structurées, dans une enceinte non pas constituée comme traditionnellement d'un haut mur mais d'une charmille ${ }^{16}$, Auzelle dessine différents espaces d'inhumation, entourés de verdure et de plantations les isolant les uns des autres, sur la base d'une trame orthogonale générale de $7 \times 7 \mathrm{~m}$. Cette variété d'espace répond à sa volonté de proposer également des modes d'inhumation variés, sépultures, enfeus, ossuaires. Les surfaces de $49 \mathrm{~m}^{2}$ ainsi délimitées peuvent recevoir dix, cinq ou deux tombes disposées de manière variée. Les bandes de $7 \mathrm{~m}$ servant à séparer les aires d'inhumation peuvent être soit des levées de terre pour des talus plantés, soit des murs de béton le long desquels s'adossent des tombes, soit des enfeus en rangées, soit des portiques à enfeus (le programme autorisant trois hauteurs maximum de cercueils). Les arbres de haute tige sont placés en périphérie de l'équipement, comme les espaces d'inhumation en sépultures isolées. Des alignements de peupliers d'Italie déterminent neuf vastes aires d'inhumation carrées (carrés cernés de ronds noirs sur le plan). Des enfeus sous portiques délimitent quatre autres aires d'inhumation quadrangulaires (carrés cernés d'un double trait continu). Les ossuaires - collectifs et individuels - sont regroupés dans un carré formé de conifères (carré entouré d'étoiles au niveau de l'angle rentrant du terrain au nord) (fig. 7, 8, 9). 
Figure 7

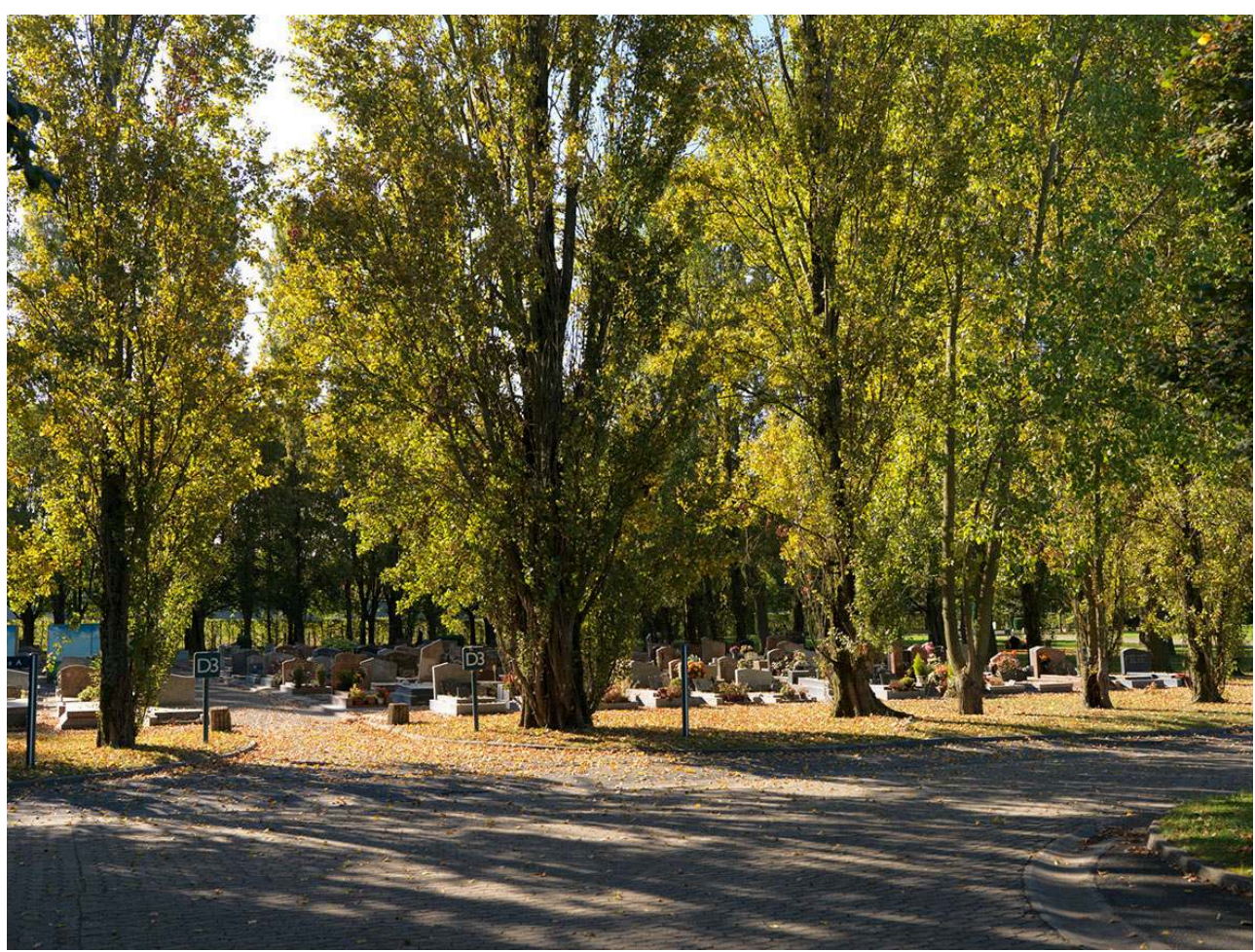

Un carré d'inhumation, bordé de peupliers.

PHOT. ASSELINE, STÉPHANE (20149400006). @ INVENTAIRE géNÉRAL DU PATRIMOINE CULTUREL. RÉgION ÎLE-DE-FRANCE, 2014.

Figure 8

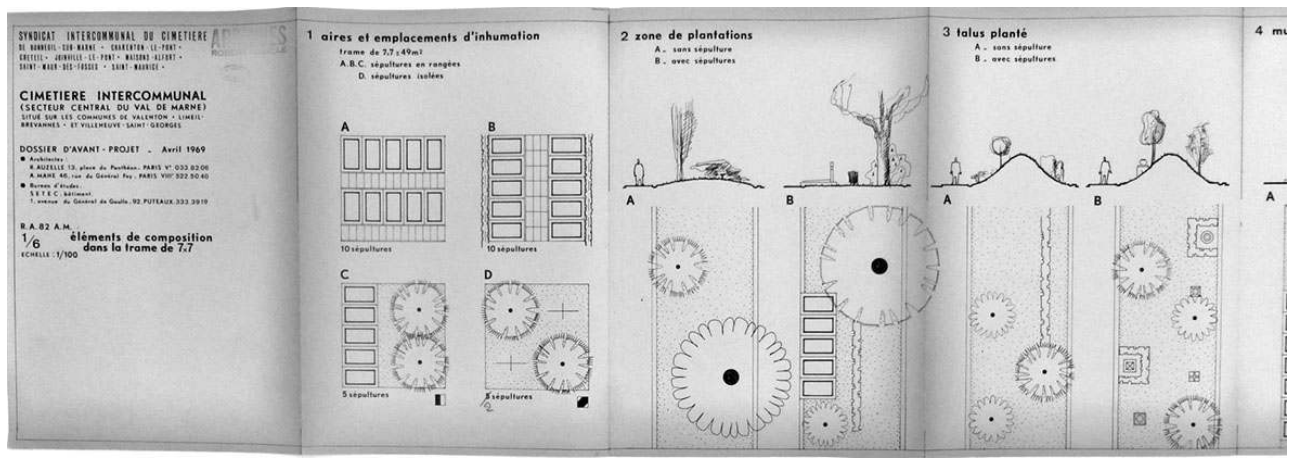

Éléments de composition dans la trame $7 \times 7$ (partie 1). Dossier d'avant-projet, avril 1969. SIAF/CAPA. Fonds Robert Auzelle 242 IFA. Carton 57, affaire RA82. En 1 les différents types d'implantation des tombes (par 10, 5 ou 2) ; en 2 les zones de plantation avec ou sans sépulture ; en 3 les talus plantés avec ou sans sépulture (aménagement non réalisé).

REPRO. DUHAU, ISABELLE (20149400031). @ INVENTAIRE gÉNÉRAL DU PATRIMOINE CULTUREL. RÉgION ÎLE-DE-FRANCE, 2014. 


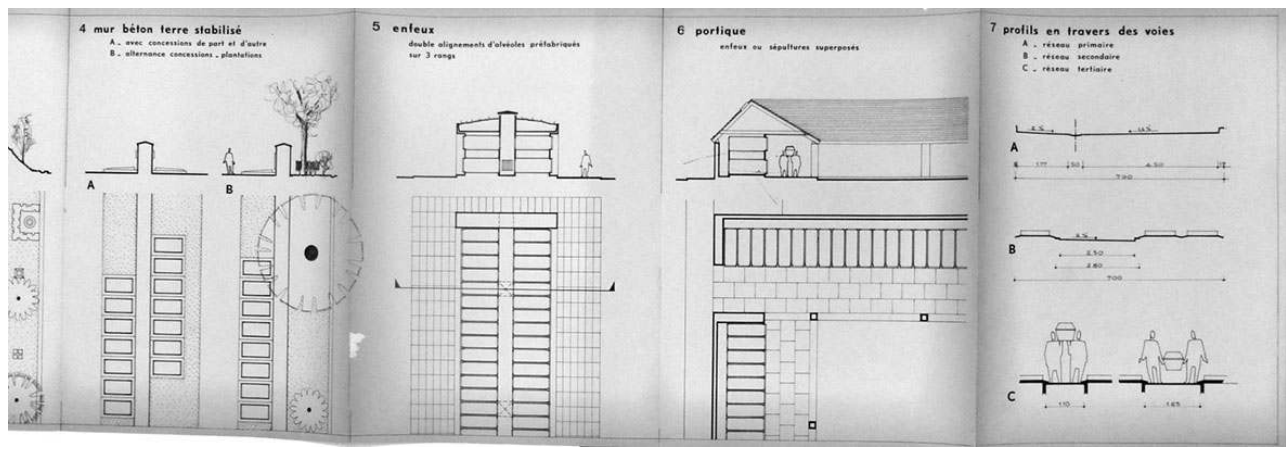

Éléments de composition dans la trame $7 \times 7$ (partie 2). Dossier d'avant-projet, avril 1969. SIAF/CAPA. Fonds Robert Auzelle 242 IFA. Carton 57, affaire RA82. En 4 les « murs béton terre stabilisé » avec sépultures de part et d'autre et sépulture d'un côté et plantations de l'autre (aménagement non réalisé) ; en 5 les enfeus ; en 6 les enfeus sous portique (les deux rangs d'enfeus superposés ont été remplacés par quatre rangs d'ossuaires individuels lors de la réalisation, eux-mêmes devenus columbariums après la création d'un crématorium); en 7 les profils des trois types de réseaux.

REPRO. DUHAU, ISABELLE (20149400032). @ INVENTAIRE gÉNÉRAL DU PATRIMOINE CULTUREL. RÉgION ÎLE-DE-FRANCE, 2014.

11 Les circulations sont hiérarchisées selon trois niveaux : un niveau primaire, destiné à la circulation automobile à deux sens, ne dessert pas directement les aires d'inhumation ; un niveau secondaire dessert, lui, les aires d'inhumation, ainsi le portage à bras des cercueils n'excède pas 30 à $40 \mathrm{~m}$; enfin, un niveau tertiaire s'adresse aux visiteurs piétons. À l'entrée le parking dispose de 550 places de stationnement ${ }^{17}$. Les bâtiments de service, localisés en position centrale, constituent l'élément majeur de la composition. Conformément au programme longuement mûri par l'architecte depuis ses premières recherches, le cimetière de Valenton comprend un espace dédié aux cérémonies afin de faciliter un rituel civil du deuil et de l'inhumation qui ne passe plus nécessairement par l'Église ${ }^{18}$. L'ensemble des bâtiments se décomposent ainsi en :

- une « aire de cérémonie » (A) desservie par des rampes d'accès, volume surélevé pour permettre la réalisation d'un funérarium en demi sous-sol ;

13 - un portique (B) servant d'entrée principale ;

14 - un bâtiment de la conservation (C) (comprenant également deux studios);

15 - un bâtiment (D) destiné à cinq commerces en RDC et logements des commerçants à l'étage ;

16 - un bâtiment (E), abritant locaux du fossoyeur, des jardiniers, hangar et ateliers ;

17 - un parterre gazonné (F) pour la dispersion des cendres (dans lequel Auzelle réservait un emplacement pour un éventuel futur four crématoire F').

18 - une longue bande de $7 \mathrm{~m}$ de large mise en eau, destinée à un ensemble sculpté monumental (G) (fig. 10). 
Figure 10

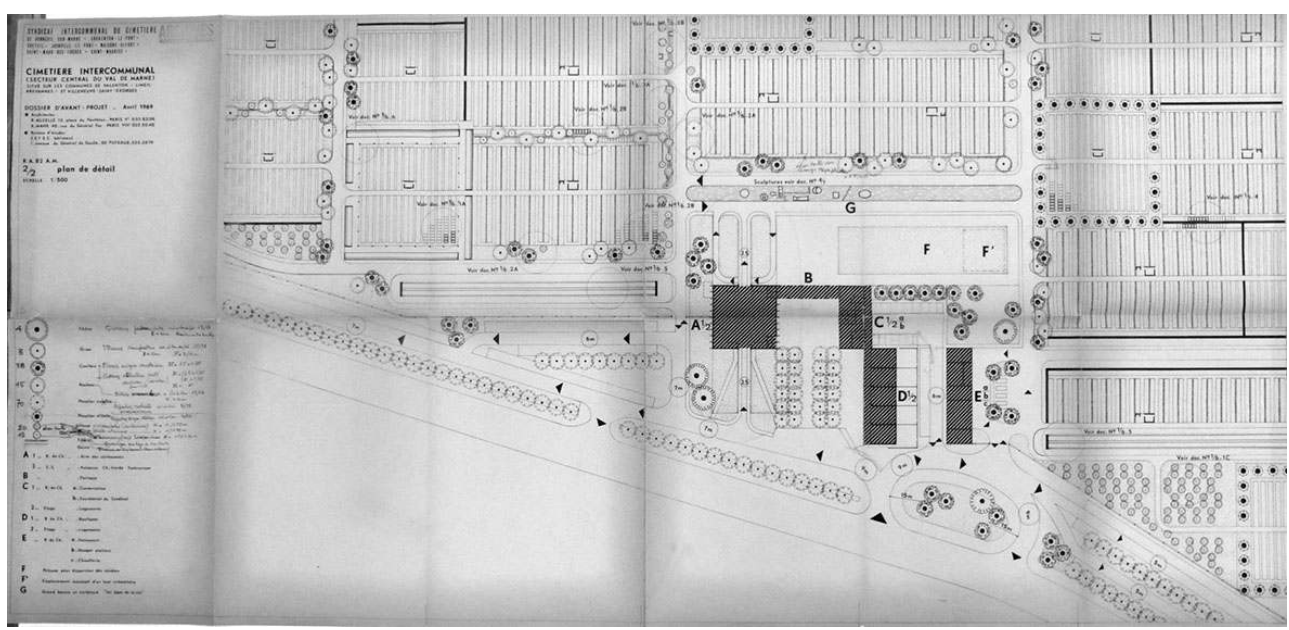

Plan de détail (en noir les bâtiments de service). Dossier d'avant-projet, avril 1969. SIAF/CAPA. Fonds Robert Auzelle 242 IFA. Carton 57, affaire RA82.

REPRO. DUHAU, ISABELLE (20149400030). @ INVENTAIRE gÉNÉRAL DU PATRIMOINE CULTUREL. RÉgION ÎLE-DE-FRANCE, 2014.

\section{Le cimetière réalisé}

Dans la foulée des études, les travaux d'aménagement et de construction se déroulent en deux phases; le cimetière ainsi que ses divers équipements ouvrent progressivement entre 1973 et 1976. Durant le chantier, certaines modifications sont apportées à l'avant-projet, pour des raisons économiques mais également pour satisfaire aux demandes des partisans d'une conception plus traditionnelle du cimetière français ${ }^{19}$. Auzelle, par volonté d'économiser l'espace occupé par les aires d'inhumation au bénéfice de zones paysagères, avait prévu beaucoup de places d'enfeus, dispositif peu prisé des familles. Le syndicat demande que le nombre de ces enfeus soit restreint; ceux disposés en portiques quadrangulaires sont abandonnés. Seuls sont gardés les enfeus en bande, auparavant disséminés dans toute la composition et qui sont regroupés sur un espace dallé unique (au sud des commerces). La formule des quatre portiques disposés en carré, évocation du cloître, n'est conservée qu'en un seul emplacement, celui réservé aux ossuaires individuels qu'ils abritent, tandis que quatre fosses collectives sont placées au centre de l'espace ainsi délimité. Ces portiques et les niches individuelles sont construits en béton laissé brut. Ils sont surmontés d'amples charpentes en bois lamellé-collé laissées apparentes et couvertes de toitures d'ardoise, ce dernier matériau revêtant également les édicules des fosses collectives (fig. 11, 12, 13). 
Figure 11

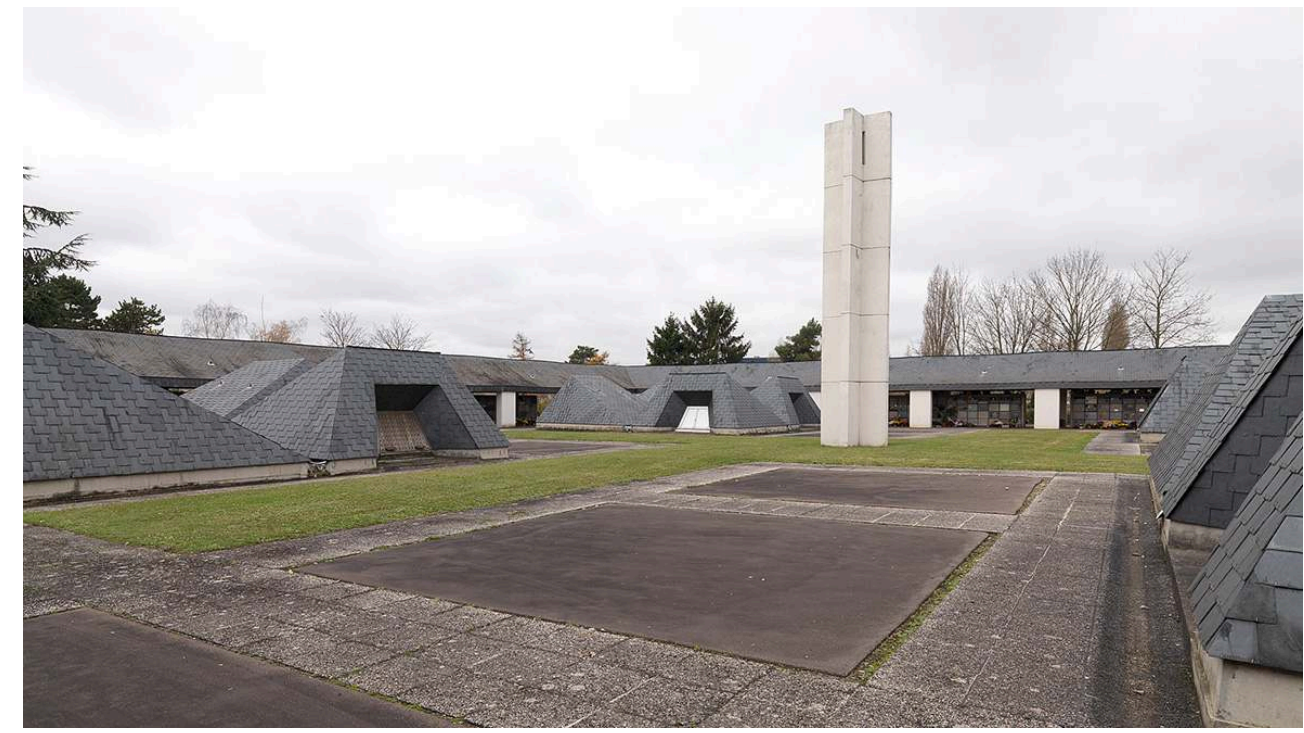

Vue d'ensemble des portiques de l'ossuaire et des quatre fosses collectives au centre.

PHOT. ASSELINE, STÉPHANE (20089400096). C INVENTAIRE gÉNÉRAL DU PATRIMOINE CULTUREL. RÉgION ÎLE-DE-FRANCE, 2008.

\section{Figure 12}

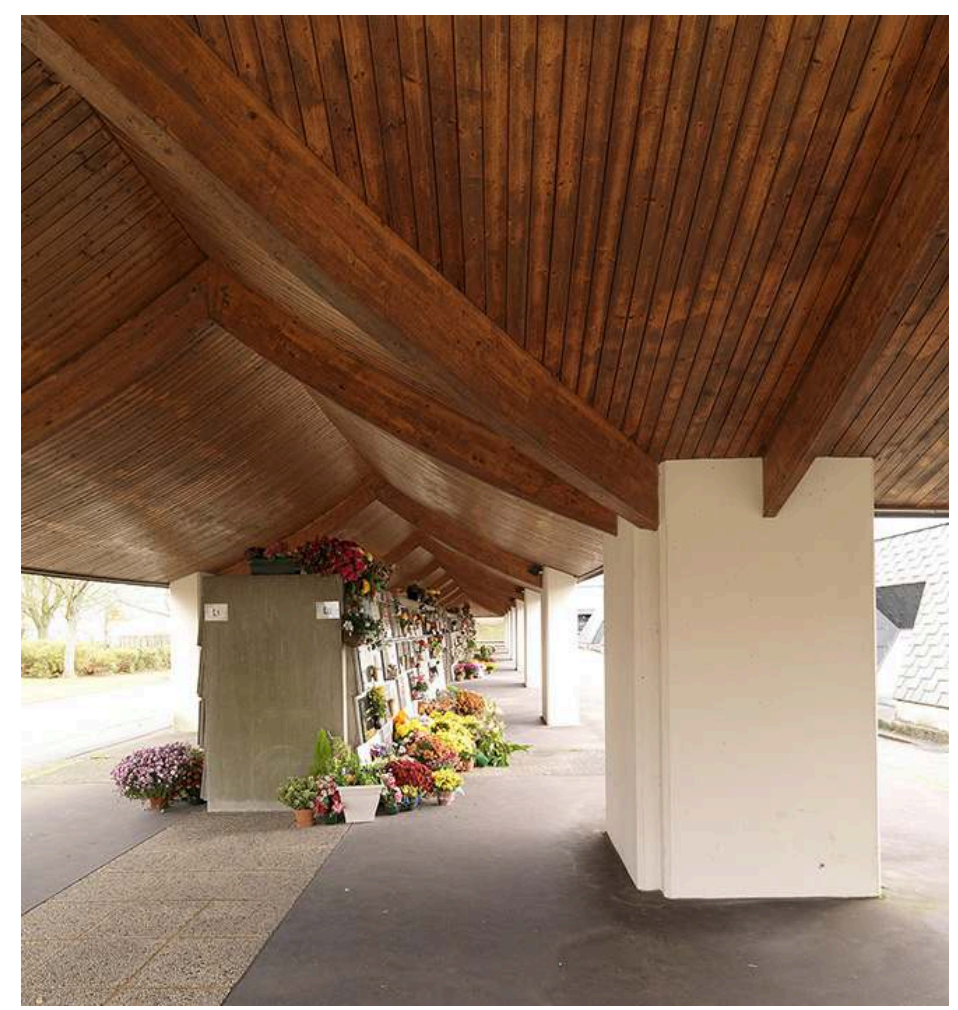

Un des quatre portiques protégeant les ossuaires individuels (aujourd'hui columbariums).

PHOT. ASSELINE, STÉPHANE (20089400098). ( ) INVENTAIRE gÉNÉrAL du PATRIMOINE CULTUREL. RÉgION ÎLE-DE-FRANCE, 2008. 


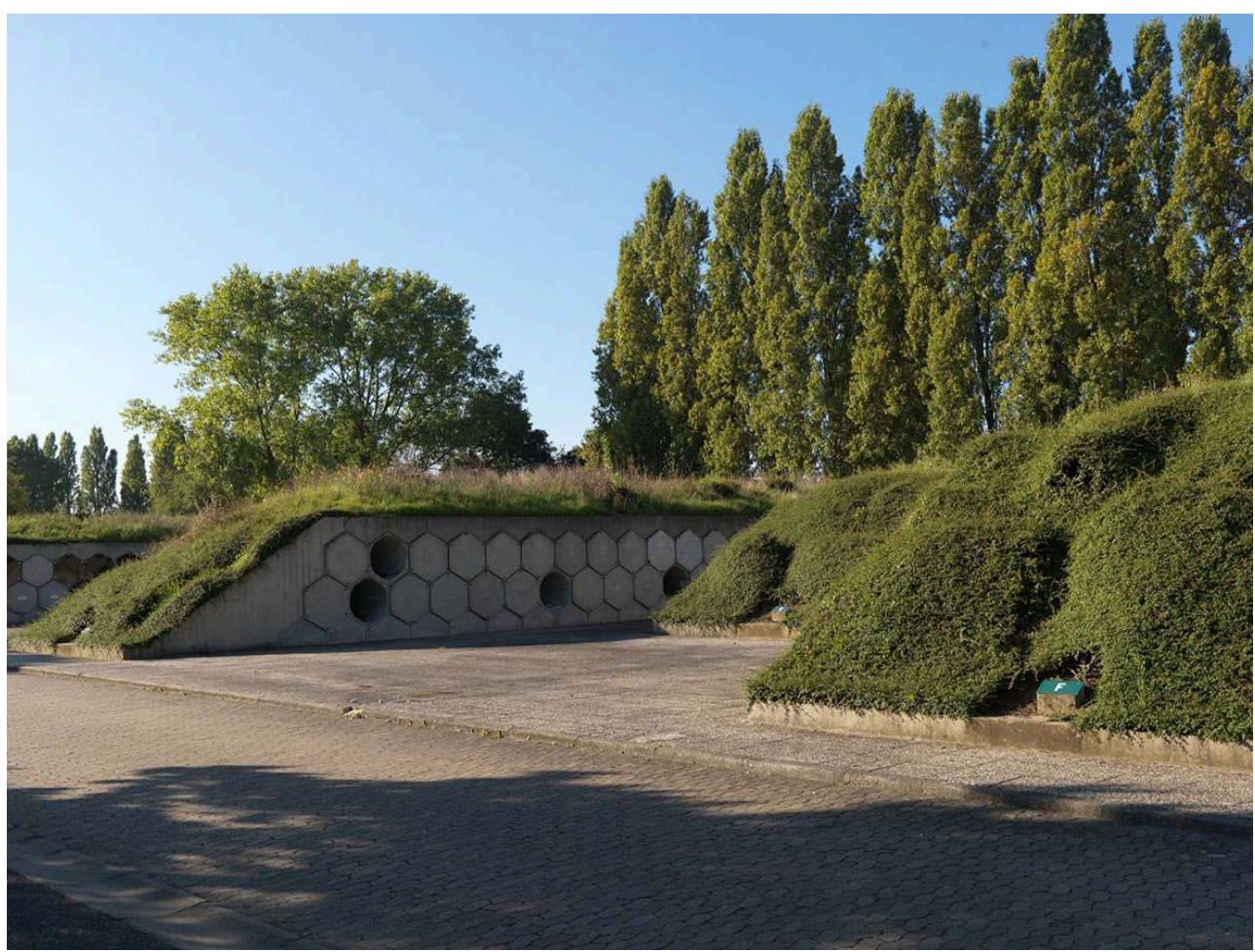

Allées d'enfeus entièrement couvertes de végétation rampante, camouflant les cheminées d'aération.

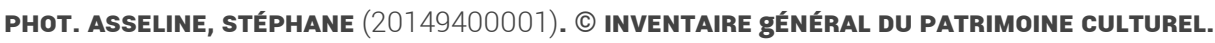
RÉgION ÎLE-DE-FRANCE, 2014.

Béton brut, ardoise et bois se conjuguent également dans la mise en œuvre des bâtiments de service. Auzelle apporte un soin particulier à leur dessin et à leur articulation, déployant deux critères qu'il estime fondamentaux : «l'harmonisation qui recherche une intégration discrète dans un cadre d'ensemble et le contraste qui accepte délibérément l'opposition et cherche à tirer un parti d'originalité ${ }^{20}$. Volumes géométriques simples sobrement construits en béton, ces bâtiments sont surmontés et reliés entre eux par de monumentales toitures courbes recouvertes d'ardoises. Le plus important, à gauche du portique d'entrée, comprend le funérarium dans un premier niveau, semi-enterré, et un second niveau destiné à l'espace baptisé «aire de cérémonie ». Celle-ci est couverte d'une spectaculaire charpente en lamellé-collé dont la forme complexe intègre un éclairage zénithal. Cette charpente est laissée apparente comme la sous-face parquetée du toit. L'extérieur ainsi que l'immense nef intérieure, divisible en deux vaisseaux grâce à un ingénieux système de cloisons escamotables, évoquent l'architecture chrétienne ${ }^{21}$. Les deux salles sont réparties de part et d'autre de la circulation axiale traversante destinée aux convois qui rejoignent, côté ville, puis quittent, côté cimetière, l'aire de cérémonie par deux longues rampes extérieures rectilignes. Surélévation, rampes imposantes et immenses portes métalliques s'escamotant totalement à l'arrivée du convoi confèrent sacralité et caractère symbolique au bâtiment. Le fond de chacune des deux nefs est en outre orné d'un autel et d'un ambon en inox dessinés sur mesure ${ }^{22}$ ainsi que d'un paravent sculpté, appelé « retable » par certains, commandé à Pierre Sabatier (fig. 14, 15, 16, 17). 
Figure 14

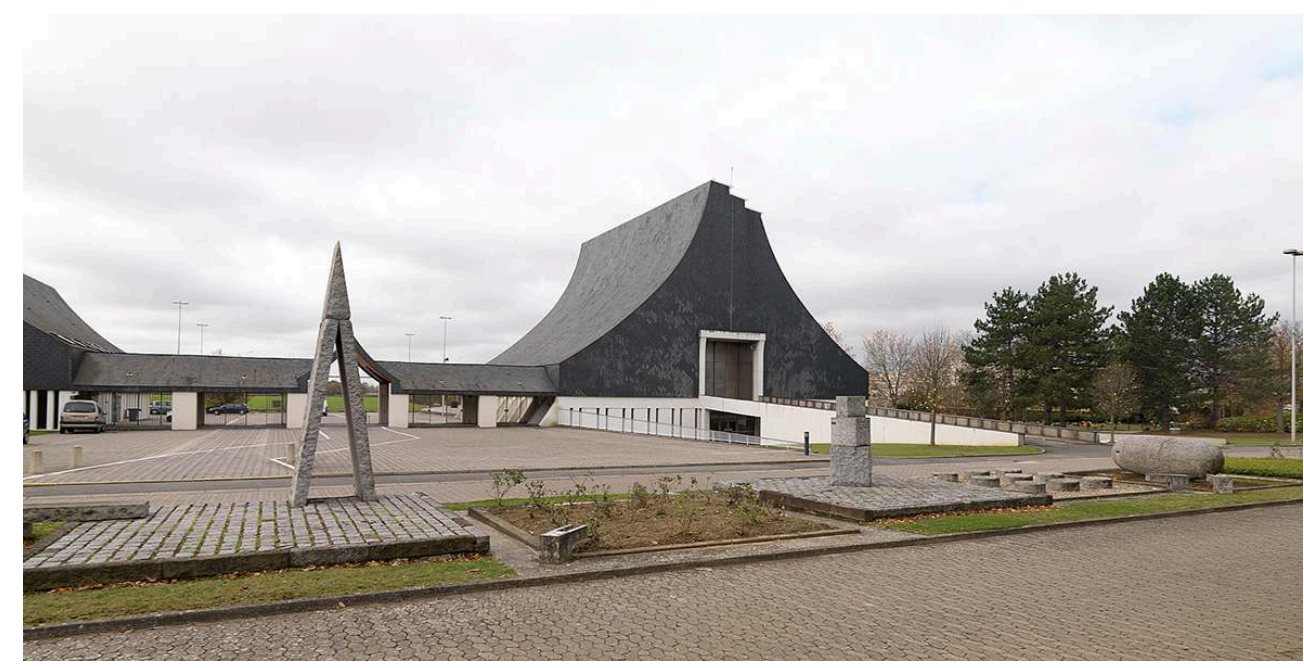

Vue d'ensemble, côté cimetière, des bâtiments d'accueil (à gauche) de l'entrée principale (au centre) et de l'aire des cérémonies (à droite) avec sa longue rampe d'accès rectiligne pour les convois. Au premier plan, la sculpture monumentale de Pierre Székely.

PHOT. ASSELINE, STÉPHANE (20089400083nuca). @ INVENTAIRE gÉNÉRAL DU PATRIMOINE CULTUREL. RÉgION ÎLE-DE-FRANCE, 2008.

Figure 15

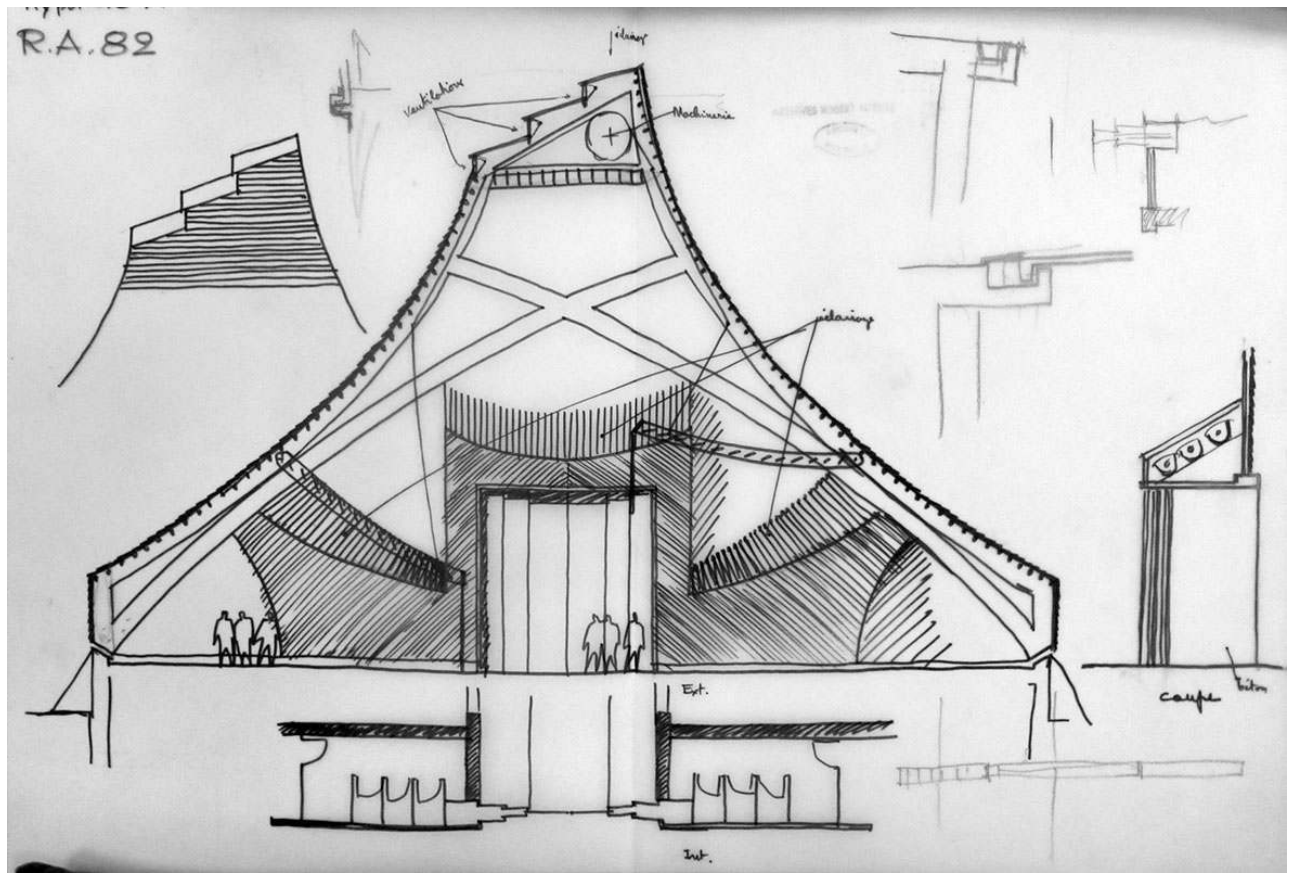

Coupe esquissée sur calque, option A, pour la salle des cérémonies, non datée, non signée (mais assurément de la main de Auzelle, son écriture et sa manière d'esquisser au feutre étant bien reconnaissables). Le système de parois mobiles, abaissées à gauche et remontées à droite, pour moduler le volume intérieur est bien visible. C'est cette option qui est retenue pour l'éclairage zénithal au faîtage de la toiture. SIAF/CAPA. Fonds Robert Auzelle 242 IFA. Carton 57, affaire RA82.

REPRO. DUHAU, ISABELLE (20149400027). @ INVENTAIRE gÉNÉRAL DU PATRIMOINE CULTUREL. RÉgION ÎLE-DE-FRANCE, 2014 
Figure 16

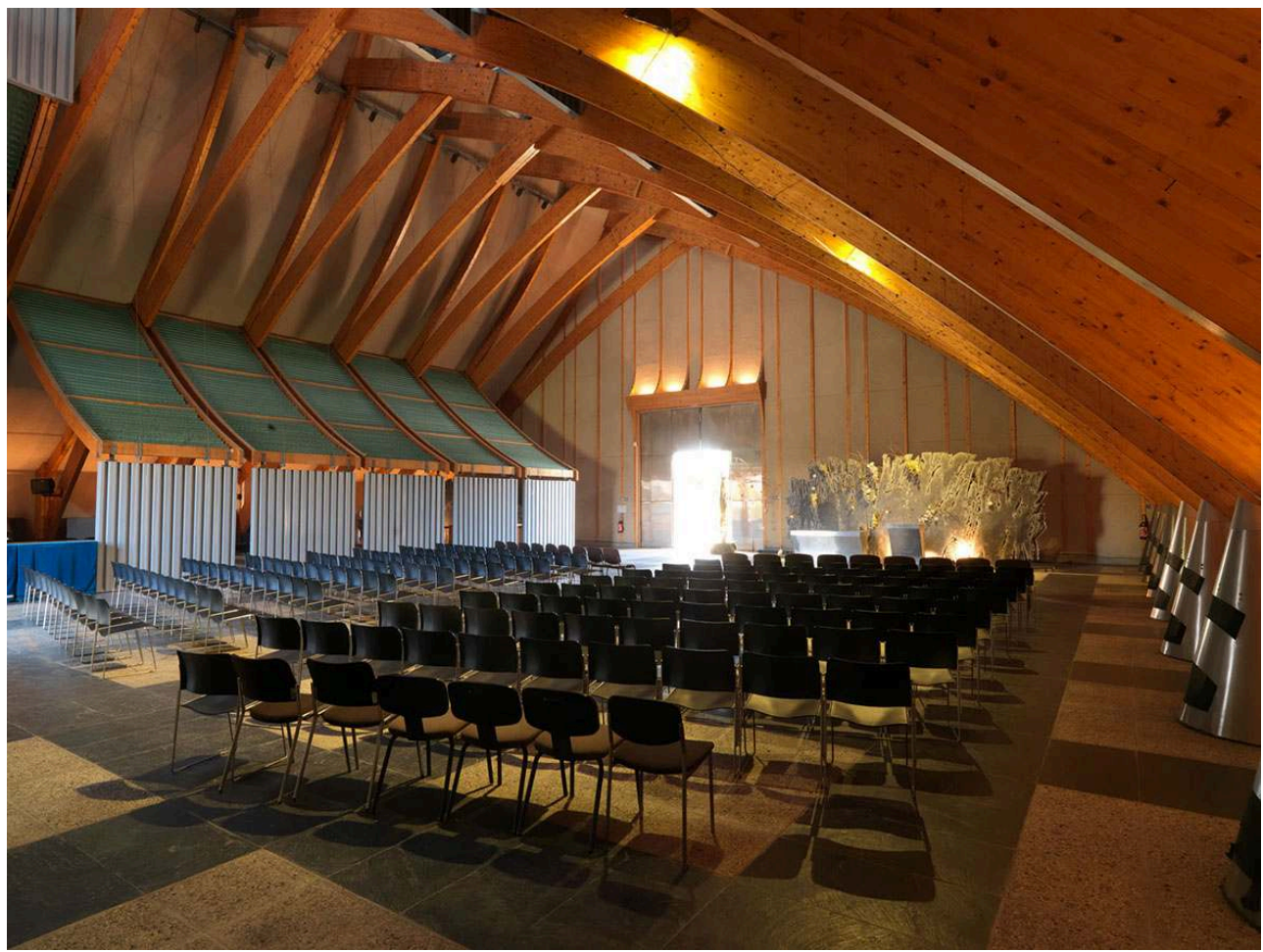

Dans cette configuration, l'immense nef de l'aire de cérémonies est réduite grâce à l'abaissement de cinq des six parois mobiles à gauche. Au fond, la porte (partiellement ouverte) permettant au convoi de quitter la salle vers le cimetière et à droite l'un des paravents de Sabatier.

PHOt. ASSELINE, STÉPHANE (20149400012nuca). @ INVENTAIRE génÉrAL DU PATRIMOINE CULTUREL. RÉgION ÎLE-DE-FRANCE, 2014. 
Figure 17

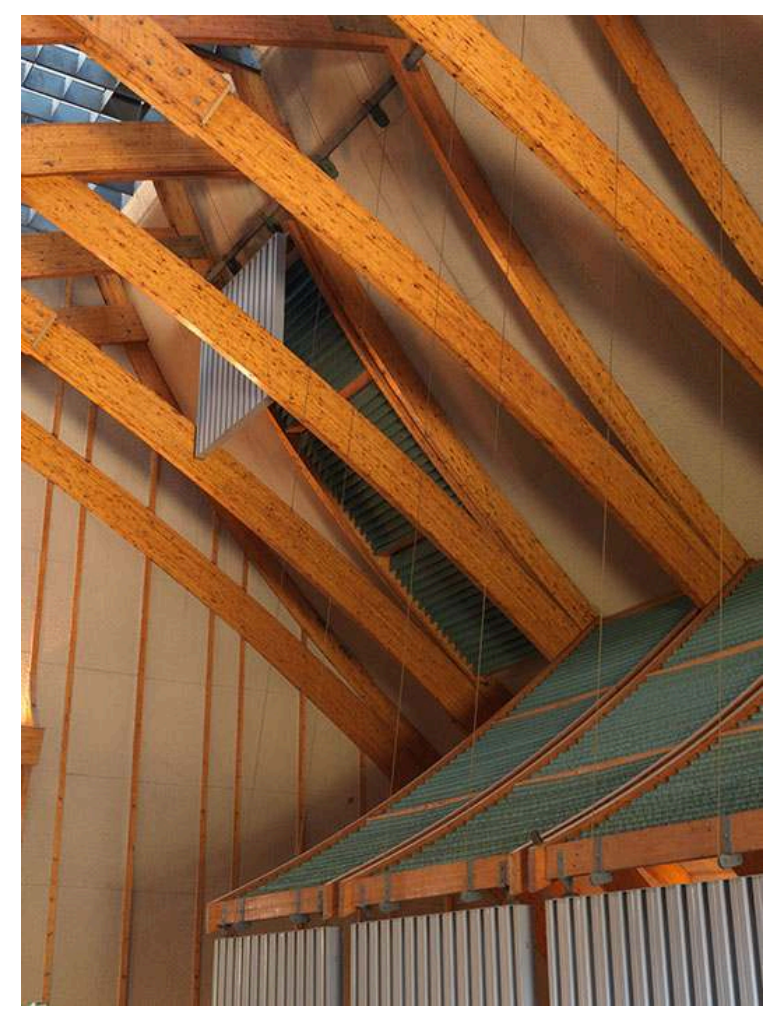

Détail de la charpente en bois lamellé-collé et du système de cloisonnement mobile.

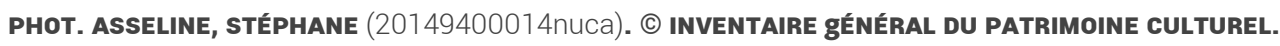
RÉgION ÎLE-DE-FRANCE, 2014.

Soucieux de faire connaître sa réalisation et de la présenter sous son meilleur jour, Auzelle fait appel aux photographes d'architecture Pierre Joly (1925-1992) et Véra Cardot (1920-2003) ${ }^{23}$ pour lui fournir les clichés qu'il distribue à son réseau et aux revues spécialisées. Il n'hésite pas à publier dans les périodiques du secteur funéraire afin de promouvoir sa conception du cimetière et de tenter de convaincre les professionnels réticents. L'usage du noir et blanc, dont la technique ne s'imposait plus en 1975, incarne parfaitement l'aspiration de l'architecte à la sobriété et à l'intemporalité, propices au recueillement et répondant à un besoin de méditation sur la mort qui, à ses yeux, ne serait pas incompatible avec le bonheur mais en serait le fondement le plus sûr (fig. 18). 


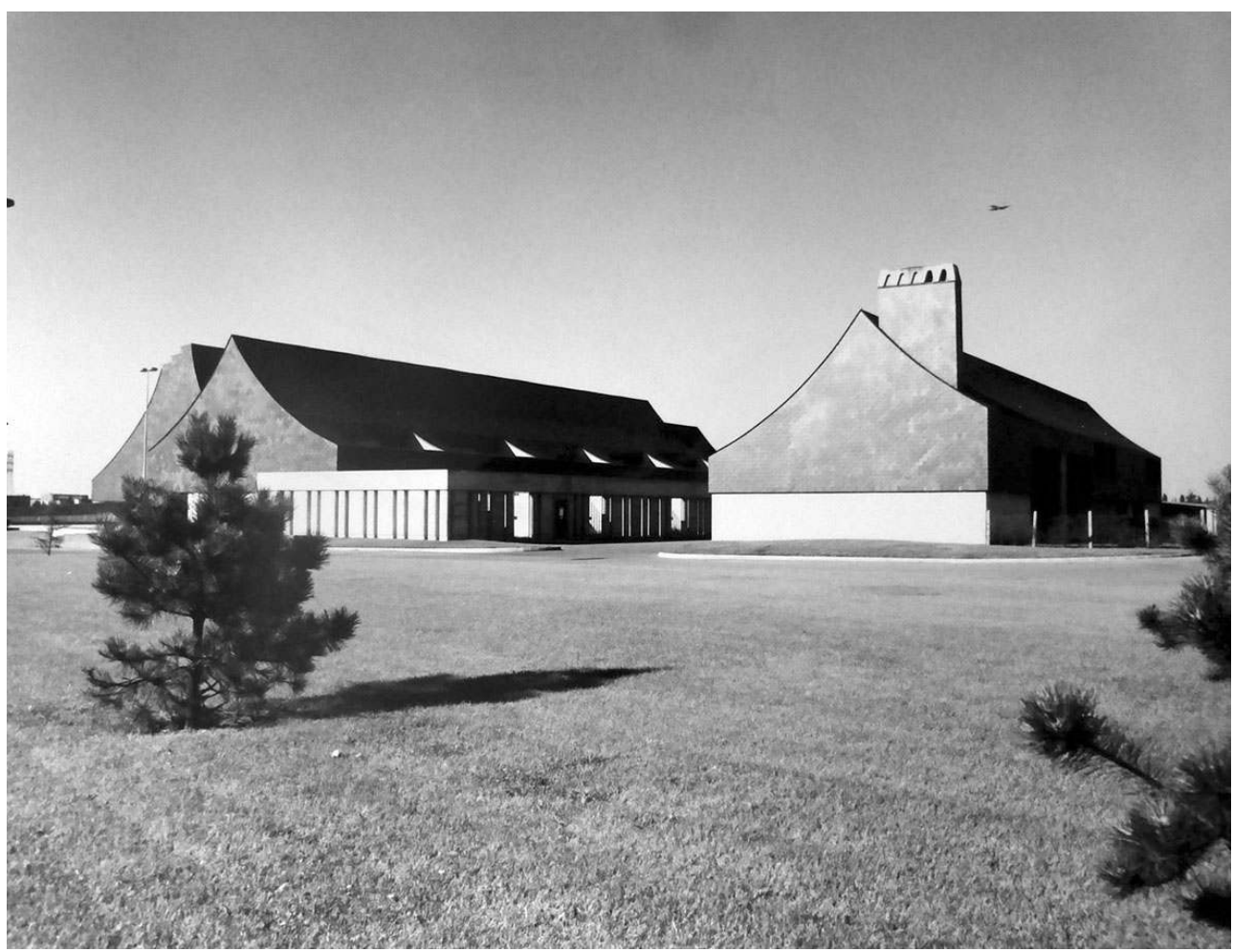

Vue générale de l'aire de service depuis l'extérieur du cimetière, à gauche le bâtiment des commerces et à droite celui des ateliers et hangars. Reproduction d'un tirage N/B signé Pierre Joly et Véra Cardot, photographes [1973]. SIAF/CAPA. Fonds Robert Auzelle 242 IFA. Carton 57, affaire RA82.

REPRO. DUHAU, ISABELLE (20149400040). @ INVENTAIRE gÉNÉRAL DU PATRIMOINE CULTUREL. RÉgION ÎLE-DE-FRANCE, 2014.

\section{Les collaborations artistiques pour une œuvre totale}

Auzelle, dès son avant-projet, prévoit un bassin accueillant un programme sculpté monumental, dans l'axe de l'entrée de la composition. Il en choisit et le thème, les âges de la vie, et l'artiste, Pierre Székely (1923-2001) ${ }^{24}$. Les deux hommes se connaissent ; ils ont déjà collaboré, Székely ayant réalisé un ensemble de sculptures/jeux d'enfants pour la cité de la Plaine à Clamart en $1957^{25}$. L'artiste ${ }^{26}$, préoccupé de recherches architecturales, adhère à l'idée de synthèse des arts qui associe architectes, peintres et sculpteurs. Il est d'abord membre du groupe Espace, fondé par André Bloc en 1951, puis du Groupe international d'architecture prospective (GIAP), fondé en 1965 par Michel Ragon. Au moment de sa conception, en 1972, le projet de Valenton est d'abord remis en cause pour des raisons financières puis finalement modifié. Auzelle réussit à convaincre le syndicat, soucieux d'une part de ne pas faire exploser le budget, mais également sceptique face au sujet de l'œuvre projetée. L'ensemble sculpté initial consistait en un long bassin rectangulaire $(160 \times 7 \mathrm{~m})$ dans lequel seraient répartis les différents éléments du programme : la naissance, l'enfance, l'adolescence, l'âge adulte, le couple, l'âge mur, la mort. Les formes étaient prévues pour émerger de l'eau et devaient être réalisées en béton par coffrage en polystyrène expansé sculpté. Les piétons auraient pu circuler entre les sculptures au moyen de massifs en béton armé à fleur du niveau de l'eau évoquant les pas des jardins japonais. 
Figure 19

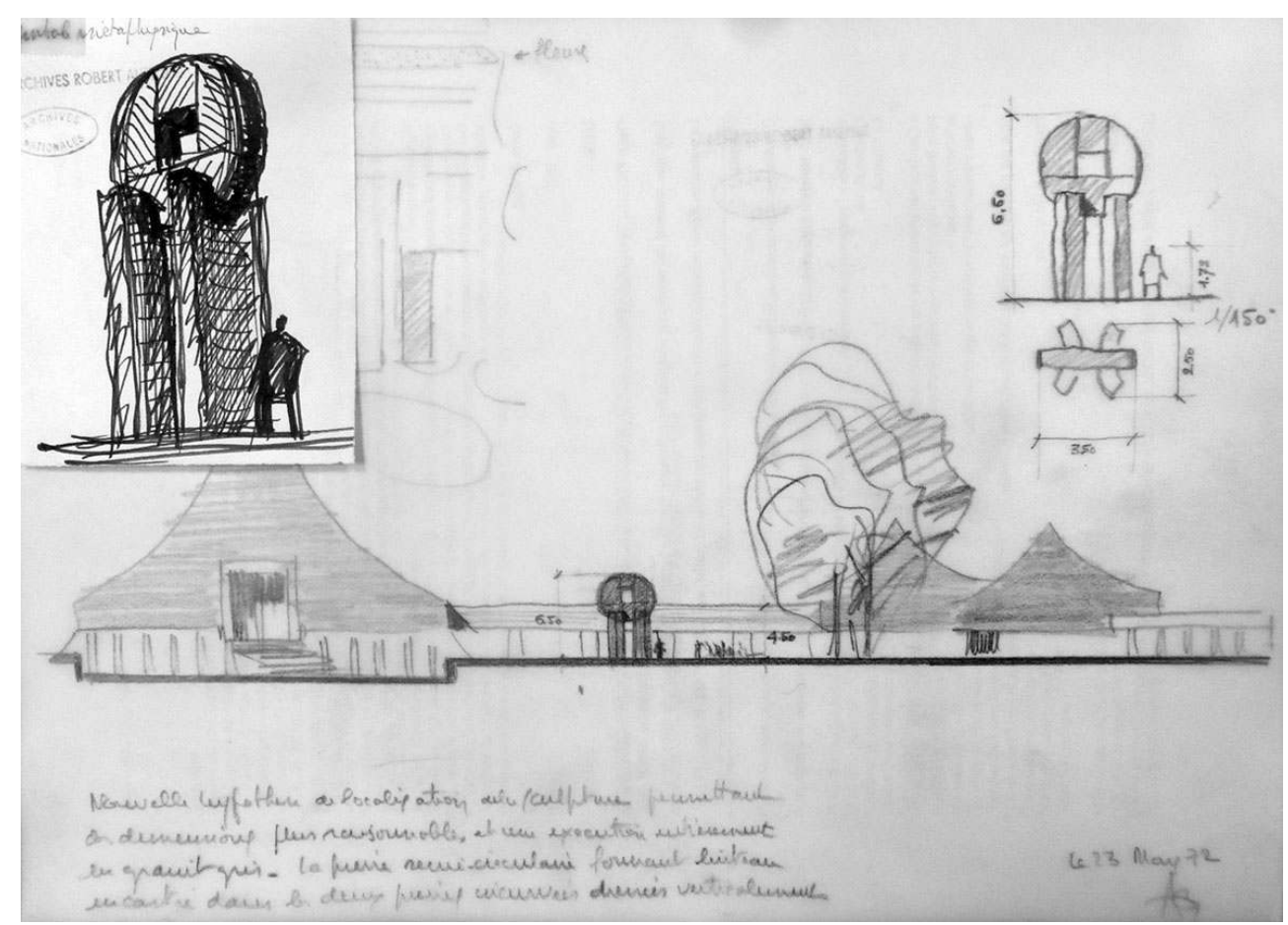

Esquisse proposant une autre sculpture monumentale, située celle-ci sur le parvis d'entrée au cimetière. Signée Robert Auzelle et datée 23 mars 1972. SIAF/CAPA. Fonds Robert Auzelle 242 IFA. Carton 57, affaire RA82.

REPRO. DUHAU, ISABELLE (20149400046). @ INVENTAIRE gÉNÉRAL DU PATRIMOINE CULTUREL. RÉgION ÎLE-DE-FRANCE, 2014.

Trop coûteux, ce premier projet doit être abandonné. Auzelle réfléchit à d'autres solutions, esquissant notamment une sculpture monumentale sur l'esplanade d'accès au cimetière (fig. 19). Finalement, à l'emplacement du bassin initial, il dessine une terrasse pavée (en granit breton du Coglais ${ }^{27}$ ) sur laquelle sont implantés des parterres carrés $(4,5 \times 4,5 \mathrm{~m})$ où alternent petits bassins, massifs plantés et éléments sculptés. Székely y conçoit « le jardin de la méditation de la vie » appelée aussi «le jardin de la médiation des âges de la vie». Treize étapes se succèdent dans le dépouillement d'un lent cheminement propice à cette méditation (fig. 20) :

- L'en-deçà inconnu,

- L'œuf de la conception

- Le lac de la naissance

- Les cubes de l'enfance

- La porte de la connaissance de soi

- Le pont de l'âge adulte

- Le banc de la collectivité

- Le siège de l'individualité

- Les marches vers/de la connaissance du monde

- La rencontre (ou le couple)

- La conscience de l'âge mûr

- La fenêtre vers la mort

- L'au-delà inconnu 
Figure 20

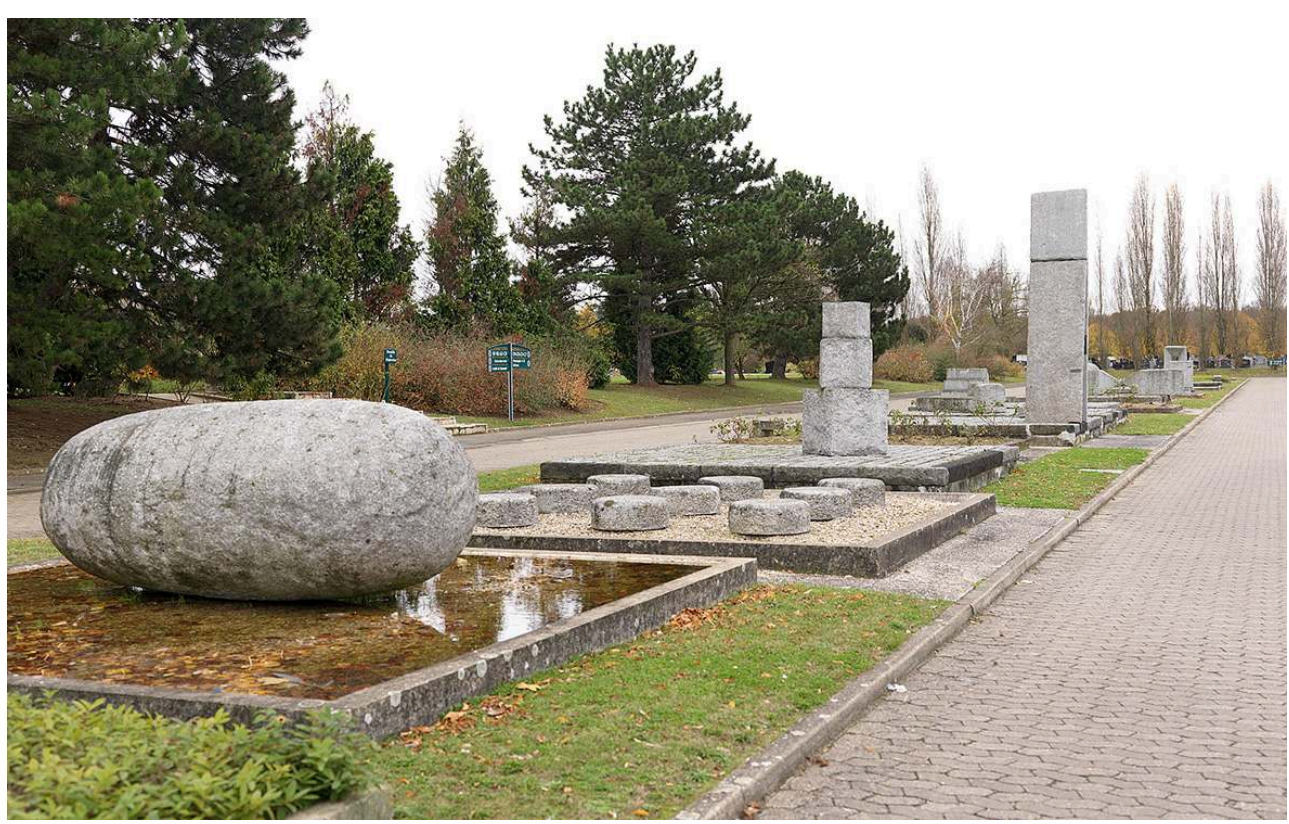

Au premier plan à gauche, «l'œuf de la conception », puis les neuf plots du « lac de la connaissance » puis « les cubes de l'enfance », enfin, élément le plus haut, « la porte de la connaissance de soi ».

PHOT. ASSELINE, STÉPHANE (20089400088nuca). @ INVENTAIRE gÉNÉRAL DU PATRIMOINE CULTUREL. RÉgION ÎLE-DE-FRANCE, 2008.

L'œuvre, en granit taillé, est réalisée par l'entreprise Bertrand à Sens-de-Bretagne sous la direction d'Édouard Hurault. Elle est dédiée par l'artiste à la mémoire du granitier breton Armand Bertrand, décédé durant sa réalisation ${ }^{28}$. Les surfaces de la pierre sont brûlées au chalumeau de kerdane (pétrole raffiné brûlant à $2800^{\circ}$ ) afin de les rendre plus rugueuses et irrégulières ${ }^{29}$, le sculpteur souhaitant reproduire l'effet naturel de l'érosion des $\operatorname{siècles}^{30}$. La simplicité des formes confère à cette œuvre magistrale une grande force symbolique en adéquation parfaite avec la vision de l'architecte ${ }^{31}$ (fig. 21). 
Figure 21

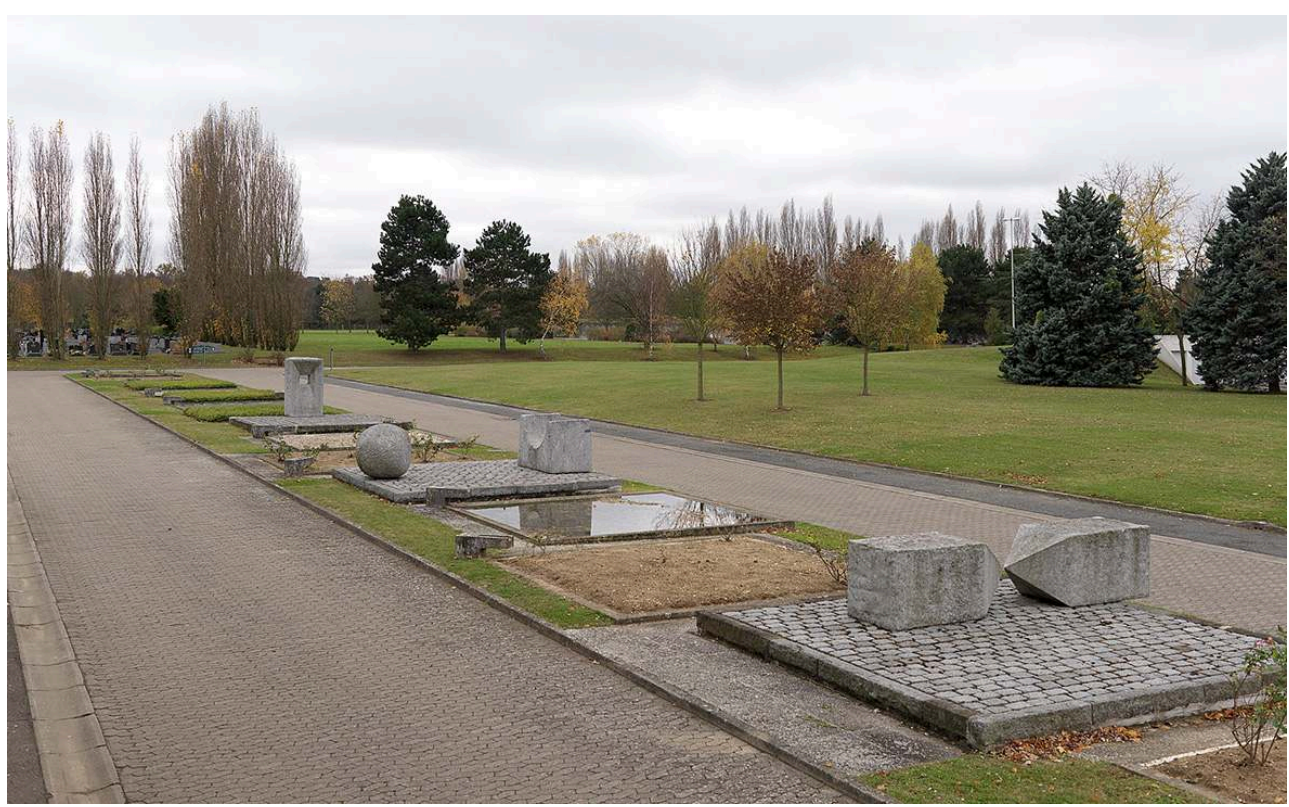

Au premier plan à droite « la rencontre », puis la « conscience de l'âge mur », la « fenêtre vers la mort », enfin les derniers bassins de «l'au-delà inconnu ».

PHOT. ASSELINE, STÉPHANE (20089400086nuca). @ INVENTAiRE gÉNÉRAL DU PATRIMOINE CULTUREL. RÉgION ÎLE-DE-FRANCE, 2008.

Cette même aspiration au recueillement et à l'introspection se retrouve dans les paravents sculptés commandés à Pierre Sabatier $(1925-2003)^{32}$ pour l'aire de cérémonie. L'artiste, adhérant au mouvement "Le Mur Vivant ${ }^{33}$ réunissant architectes et plasticiens, milite également pour l'intégration des arts dans l'architecture. Dès l'année suivante, il retravaillera avec Auzelle au cimetière de Villetaneuse, réalisant les Portes de l'au-delà. À Valenton, comme avec Székely, l'architecte choisit lui-même le thème des œuvres: des rythmes végétaux pour «l'arbre de vie » et des rythmes universels pour " le cosmos", sujets qu'il juge bien adaptés à la mixité de fonction de ces espaces. À ses yeux, ces thèmes conviennent autant aux cérémonies religieuses (la résurrection, l'immortalité dans la vie éternelle) qu'aux cérémonies civiles (le renouvellement, la renaissance de la nature et la relève de la vie) ${ }^{34}$. Sabatier réalise deux œuvres monumentales ( 7 x $3 \mathrm{~m}$ ), à l'échelle de l'architecture, selon la technique qu'il privilégie durant les années 1970, de l'étain pur travaillé sur des feuilles de laiton ajourées par fusion et aux contours déchirés. Les paravents sont installés en $1974^{35}$ (fig. 22, fig. 23). 
Figure 22

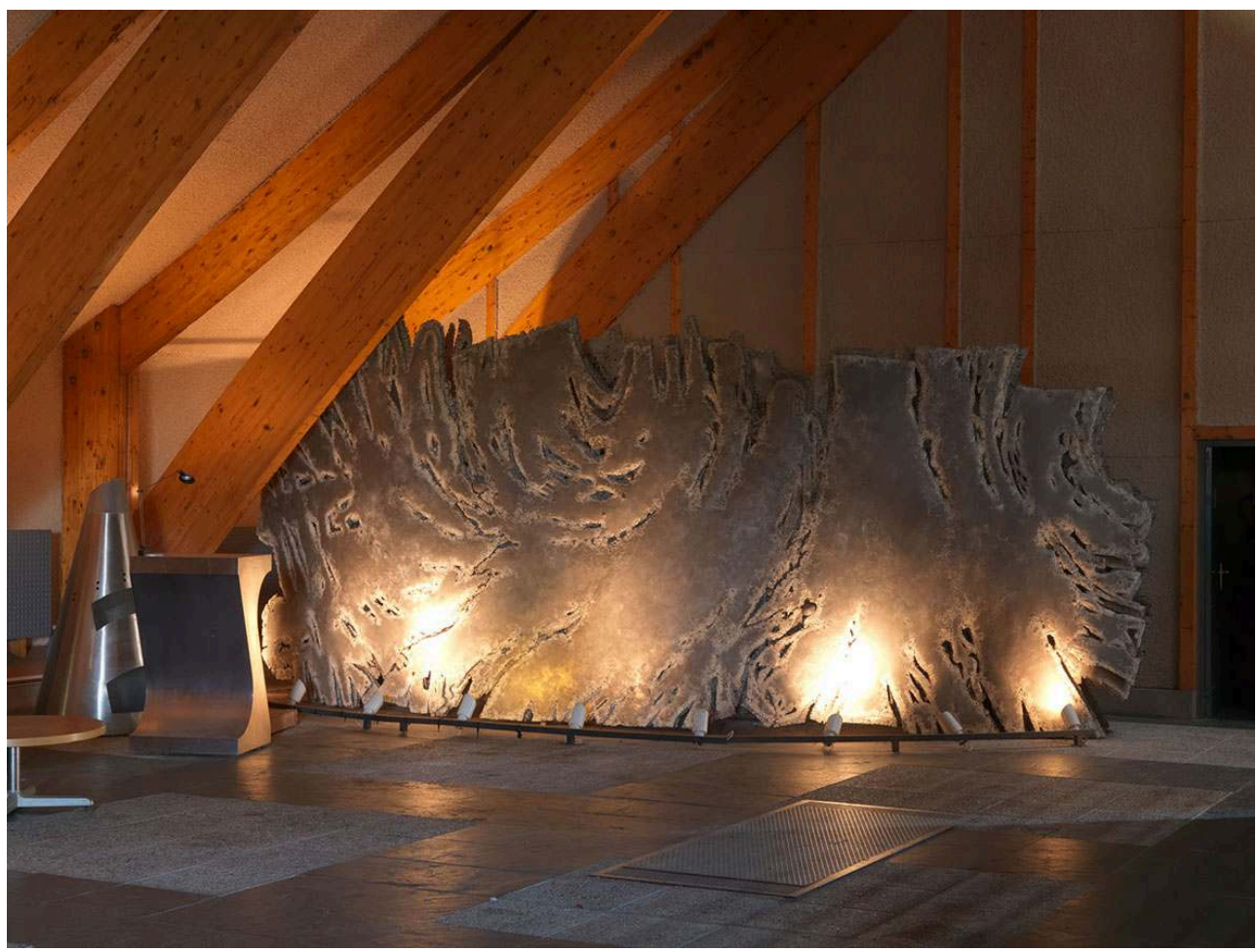

Vue d'ensemble du paravent Le Cosmos.

PHOt. ASSELINE, STÉPHANE (20149400016). @ INVENTAIRE géNÉRAL DU PATRIMOINE CULTUREL. RÉgION ÎLE-DE-FRANCE, 2014. 
Figure 23

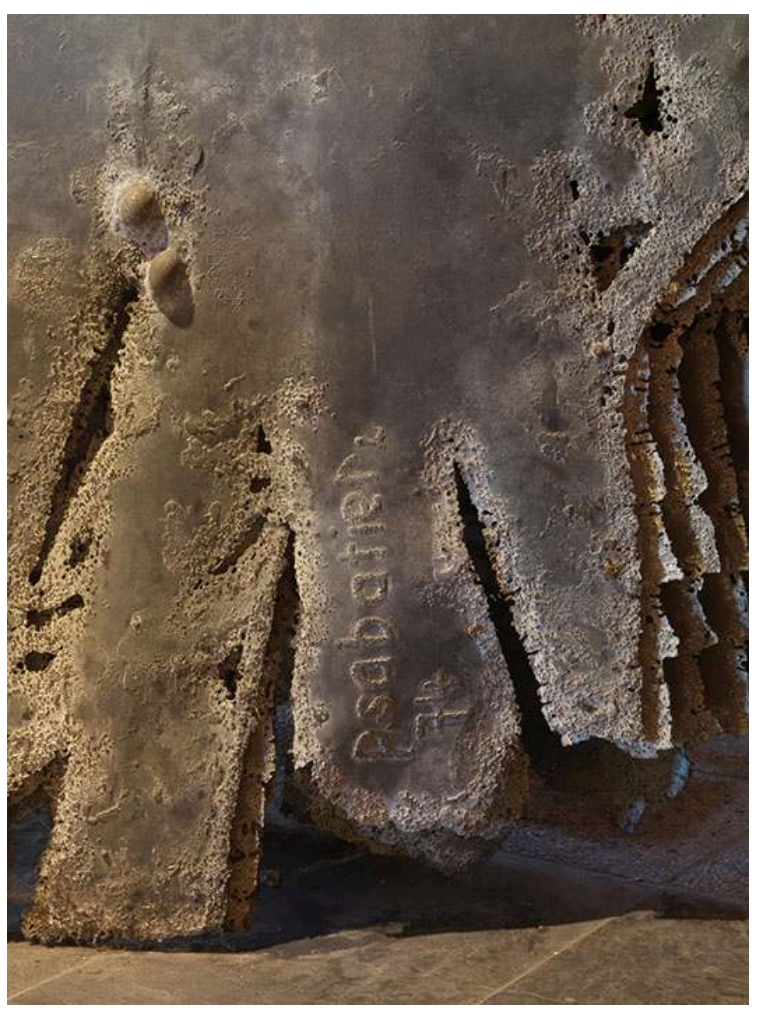

Détail de la signature de Pierre Sabatier, de la date portée sur l'œuvre « 74 » et des effets de découpe et de surface de l'étain sur les feuilles de laiton.

PHOT. ASSELINE, STÉPHANE (20149400018). @ INVENTAIRE gÉnÉRAL DU PATRIMOINE CULTUREL. RÉgION ÎLE-DE-FRANCE, 2014.

\section{Le rendez-vous manqué des sépultures}

À la recherche de la cohérence d'une œuvre globale, Auzelle dessine jusqu'aux modèles des sépultures ${ }^{36}$. Dans les premiers mois de l'ouverture du cimetière, le syndicat tente d'imposer ses dessins à l'aide d'un règlement comprenant un recueil de modèles imposés $^{37}$. L'architecte souhaite bannir les tombes monumentales et ostentatoires et réduire leur emprise au sol en en réduisant les dimensions ou en les superposant en enfeus au bénéfice des frondaisons. Maître d'ouvrage et maître d'œuvre reçoivent le soutien de la section Marbre-Granit-Pierre de la Confédération de l'artisanat et des petites entreprises du bâtiment qui visite trois des réalisations d'Auzelle et publie une brochure illustrée destinée à convaincre ses adhérents ${ }^{38}$. Pourtant, les clients, et surtout, les marbriers s'opposent rapidement à cette contrainte qui constitue pour eux à la fois une remise en cause de leurs habitudes mais aussi une éventuelle perte financière : l'architecte propose des modèles d'une grande sobriété nécessitant peu de matière première. Malgré la commande par le syndicat de quelques modèles d'exposition et de premières réalisations, le règlement est bientôt annulé, faute d'assise légale, le préfet ayant signifié son illégalitée ${ }^{39}$ (fig. 24). 
Figure 24

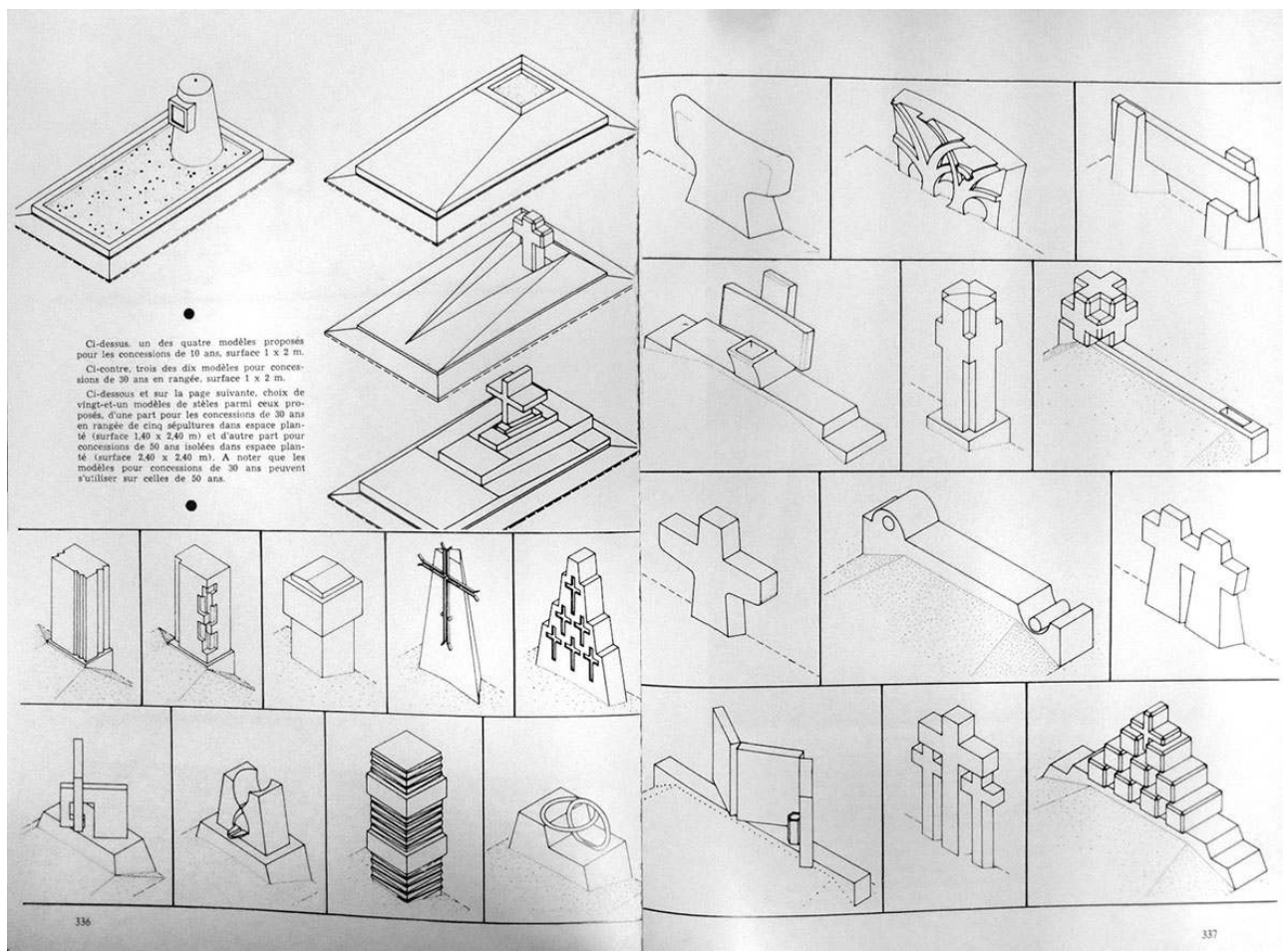

Différents modèles de sépultures tirés de l'album des modèles du cimetière de Valenton réalisé par Auzelle. Dans : MOTINOT, R. «Trois cimetières de l'île-de-France, Montfort-l'Amaury - Clamartintercommunal - Fontaine-Saint-Martin ». Le mausolée, fév. 1973, n 438, p. 336-337.

REPRO. DUHAU, ISABELLE (20149400052). (C) INVENTAIRE gÉNÉRAL DU PATRIMOINE CULTUREL. RÉgION ÎLE-DE-FRANCE, 2014.

Les enfeus conçus par Auzelle ne connaissent pas non plus de véritable succès. Le maître d'œuvre réussit à faire installer à Valenton quelques centaines d'alvéoles préfabriquées par la société Weser ${ }^{40}$, fruit de ses recherches depuis plusieurs années ${ }^{41}$ autant sur le plan formel que d'un point de vue technique (la difficulté étant l'évacuation des gaz). Ces emplacements n'ont jamais été très prisés par les familles et servent aujourd'hui surtout de caveaux provisoires. Ce sont finalement les ossuaires individuels superposés sur quatre rangs sous les portiques qui sont le plus appréciés désormais, transformés en columbariums depuis que les Français recourent de plus en plus à la crémation ${ }^{42}$ (fig. 25, fig. 26). 
Figure 25

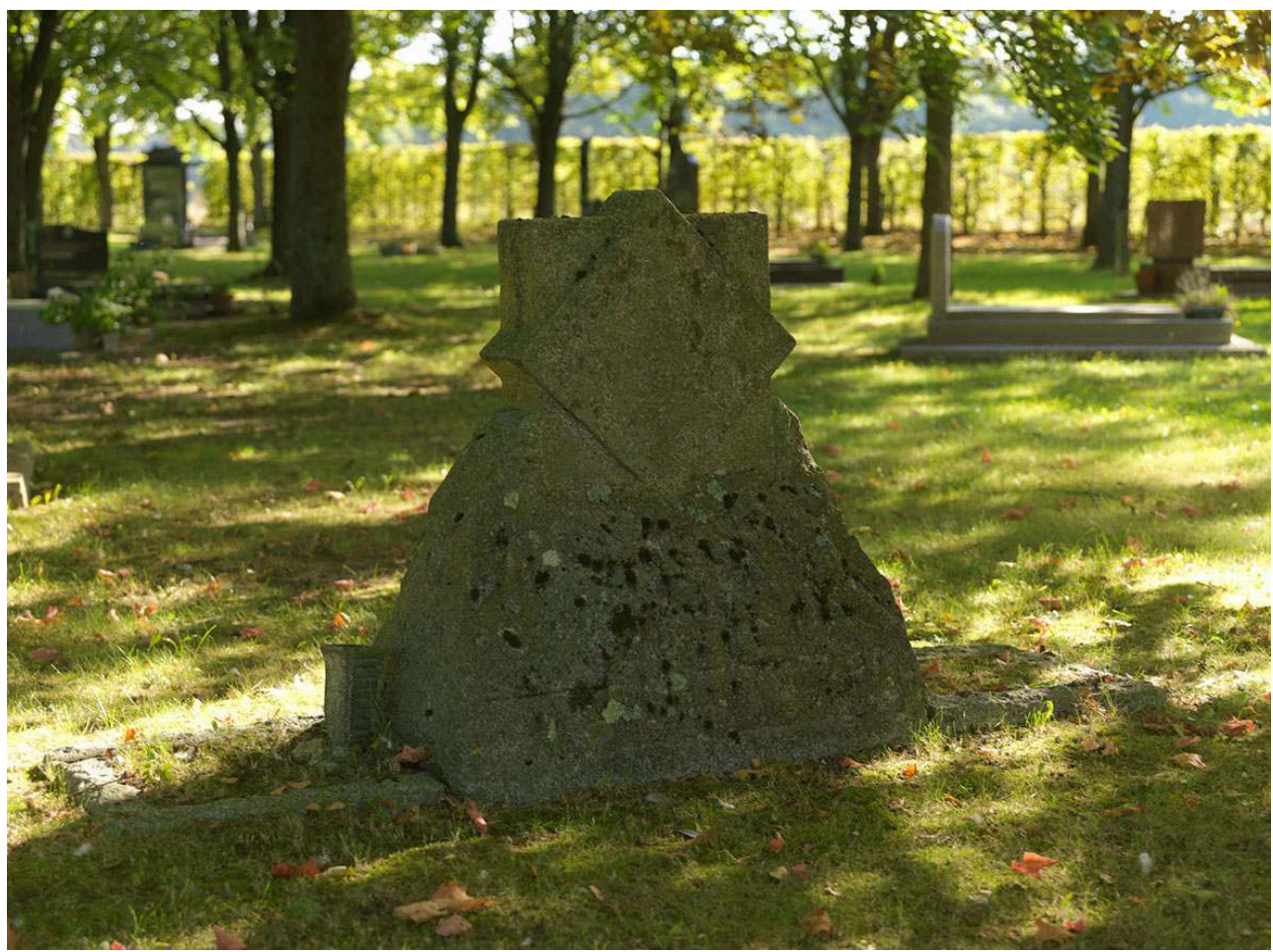

Tombe de Victor Hugo Fernandez, 1973. À l'arrière-plan, on aperçoit la haie clôturant le cimetière. PHOT. ASSELINE, STÉPHANE (20149400011). @ INVENTAIRE gÉNÉRAL DU PATRIMOINE CULTUREL. RÉgION ÎLE-DE-FRANCE, 2014. 
Figure 26

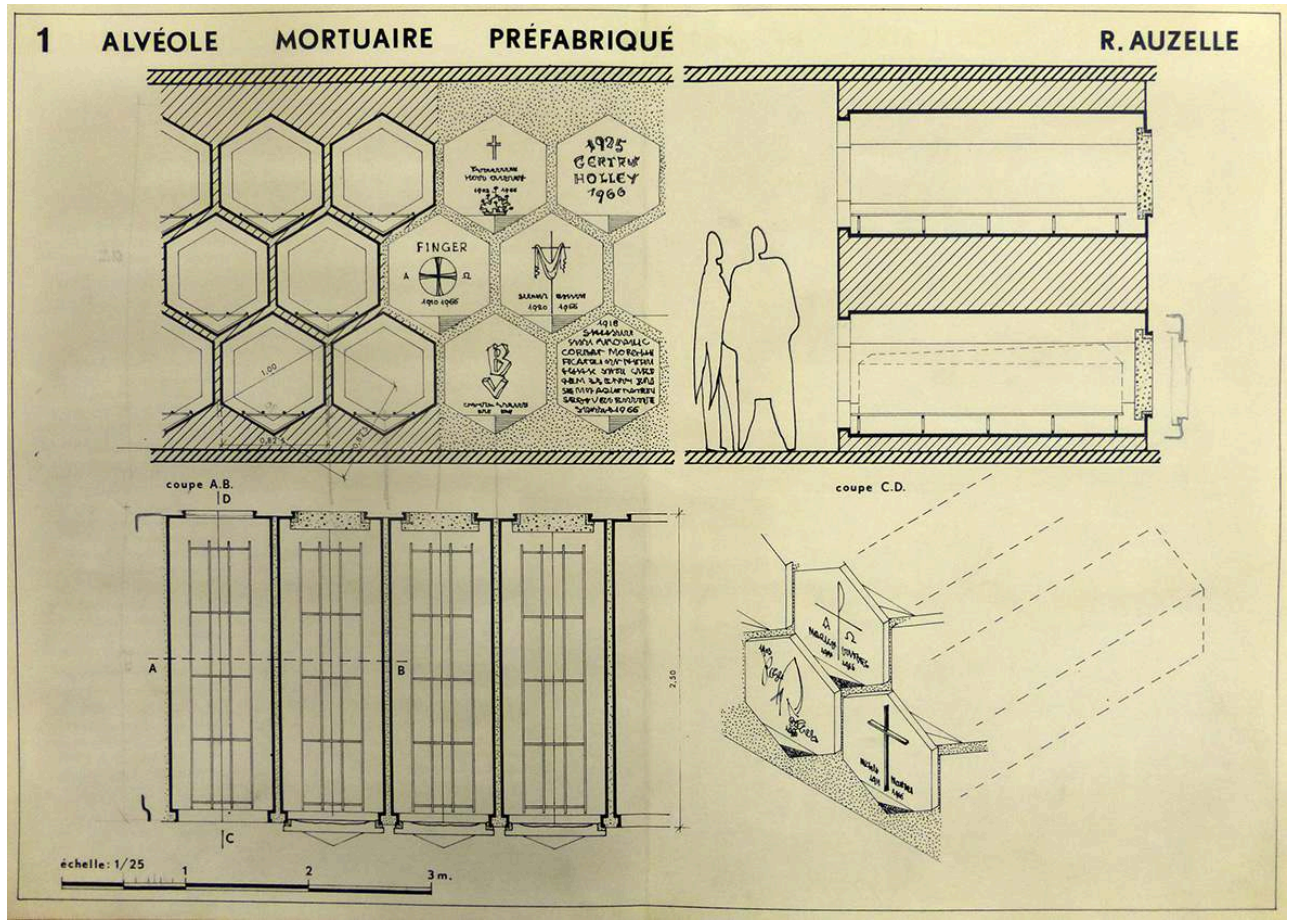

Modèle d'alvéoles mortuaires hexagonales préfabriquées pour les enfeus. Robert Auzelle (circa 1966). SIAF/CAPA. Fonds Robert Auzelle 242 IFA. Carton 61, affaire RA96.

REPRO. DUHAU, ISABELLE (P1000641). @ INVENTAIRE gÉNÉRAL DU PATRIMOINE CULTUREL. RÉgION ÎLEDE-FRANCE.

Aujourd'hui, une des plus anciennes tombes conservée dans le cimetière est celle de Victor Hugo Fernandez (4 septembre 1949-11 juillet 1973), Sud-américain décédé dans une catastrophe aérienne à Ermenonville. Sa forme géométrique, répondant aux premières exigences formelles, se voulait en outre une évocation d'un motif traditionnel inca, en référence aux origines du défunt (fig. 27). 
Figure 27

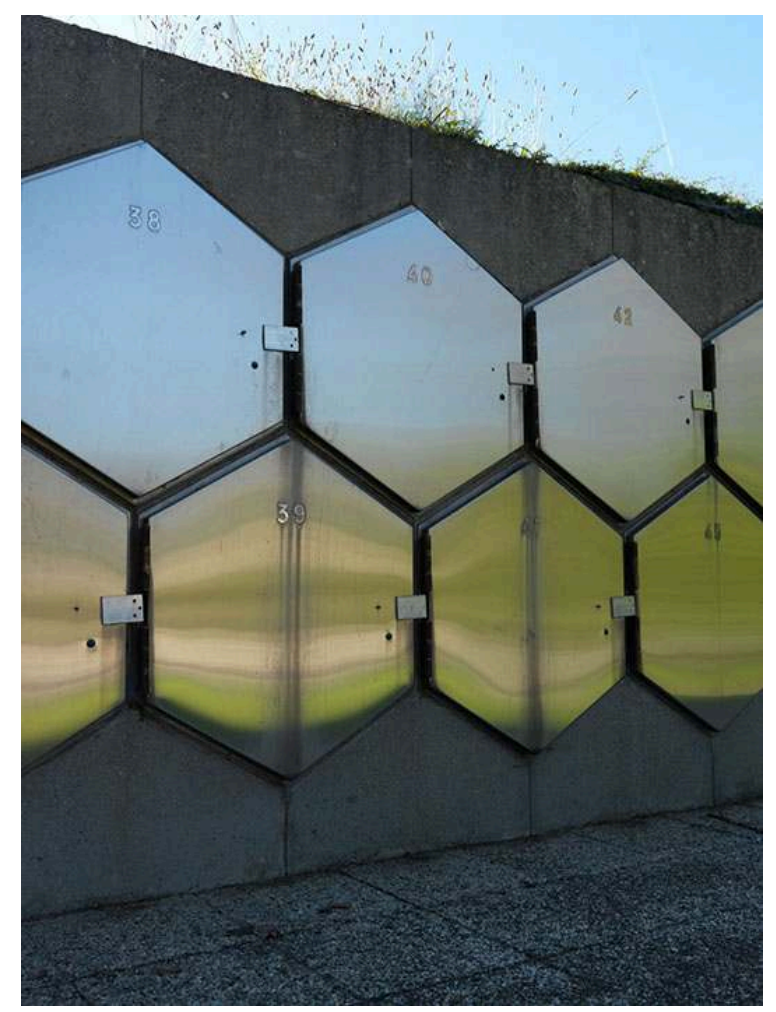

Détail des portes métalliques fermant les enfeus.

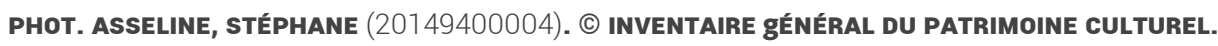
RÉgION ÎLE-DE-FRANCE, 2014.

Les carrés d'inhumation alignent quant à eux des tombeaux sans aucune particularité (quelles que soient les confessions). Les espaces paysagers accueillent des sépultures où se déploie davantage d'originalité. Cependant, leur fantaisie les éloigne tout autant de la rigueur et de la sobriété auxquelles Robert Auzelle aspirait ${ }^{43}$. Du fait de la proximité géographique de la communauté chinoise installée au sud de Paris, dans le $13^{\mathrm{e}}$ arrondissement et au-delà du périphérique, le cimetière de la Fontaine Saint-Martin comprend de nombreuses tombes asiatiques au dessin assez inhabituel dans nos nécropoles (fig. 28). Une sépulture toute récente (inhumation en 2011) attire l'attention : elle est entièrement végétale mais sa composition évoque le jardin de curé plutôt que le parterre à la française (fig. 29). 
Figure 28

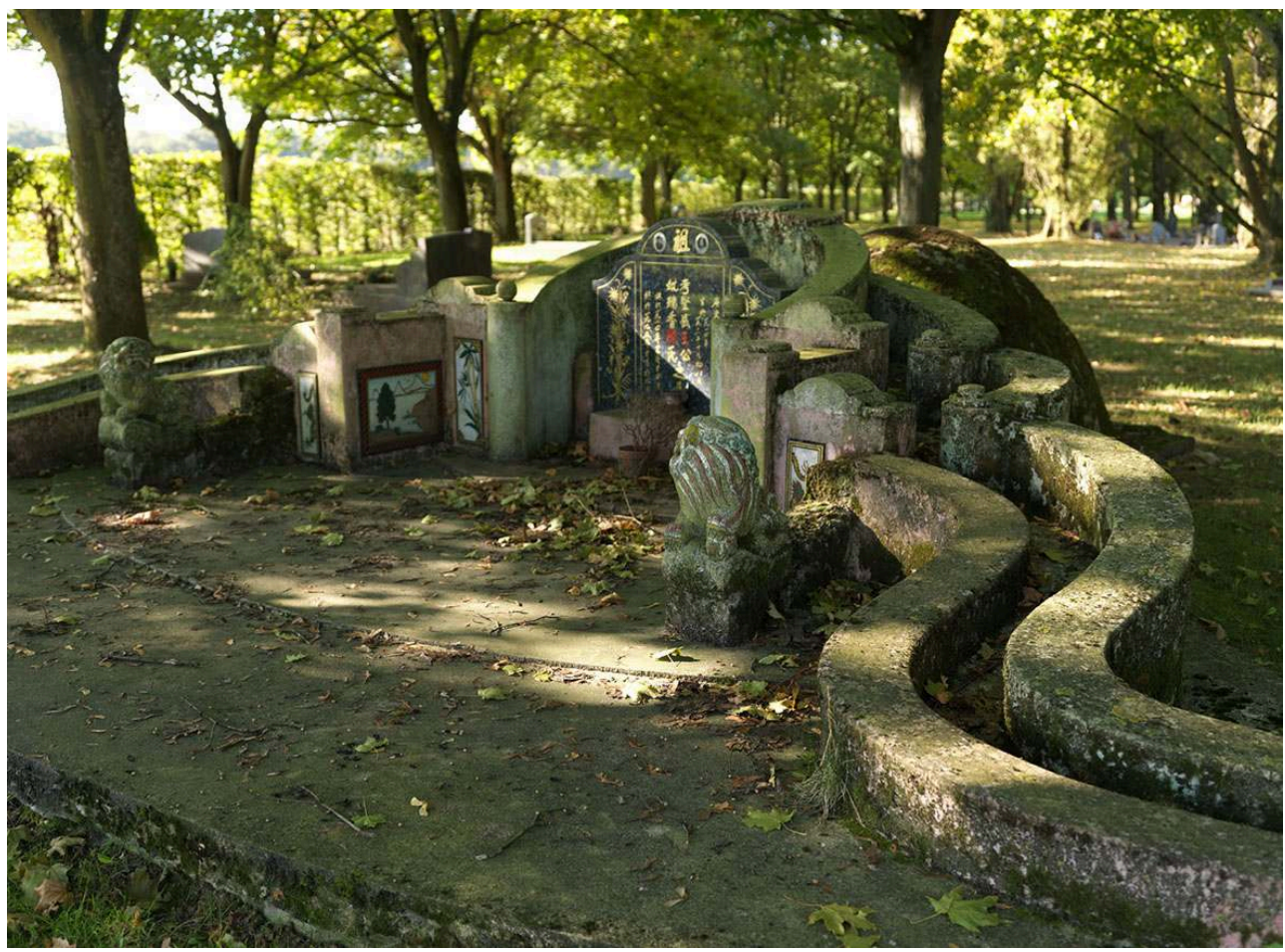

Monument funéraire d'une famille chinoise dans la zone paysagère.

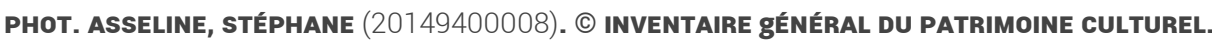
RÉgION ÎLE-DE-FRANCE, 2014. 
Figure 29

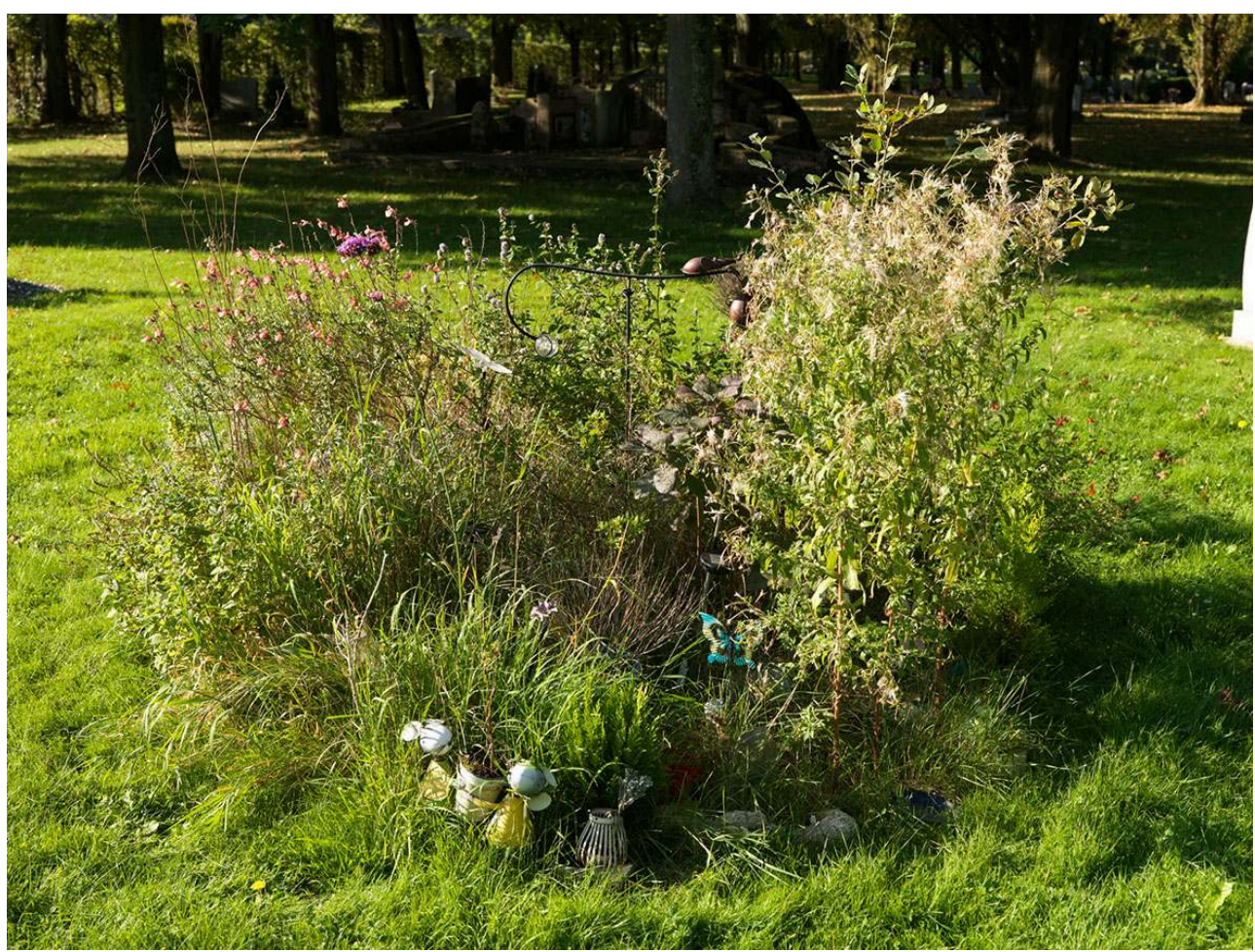

Sépulture végétale d'Yvonne Levy (1931-2011) dans la zone paysagère.

PHOT. ASSELINE, STÉPHANE (20149400009). @ INVENTAIRE gÉNÉRAL DU PATRIMOINE CULTUREL. RÉgION ÎLE-DE-FRANCE, 2014.

\section{Conclusion}

30 Le cimetière, qui s'étend sur 32 hectares $^{44}$, dispose d'un crématorium (créé en 1986 dans l'étage semi-enterré du funérarium) ${ }^{45}$, d'espaces traditionnels d'inhumation (dont un israélite et un musulman), d'espaces paysagers d'inhumation, d'un ossuaire, d'un columbarium, d'enfeus et d'un site cinéraire (avec une rocaille de dispersion, des emplacements pour des cavurnes ou cavotins ${ }^{46}$ et un jardin des innocents - espace de dispersion des cendres pour les enfants). Depuis quelques années, la meilleure gestion des cimetières municipaux des communes du syndicat (reprise de concessions abandonnées et fin des concessions à perpétuité) permet davantage d'inhumations locales; ainsi le recours au cimetière intercommunal est-il moins fréquent, environ 400 inhumations par an, et celui-ci dispose-t-il encore d'une multitude de places. Il conserve de vastes espaces libres.

31 Malgré quelques modifications par rapport à son état d'origine, le cimetière de Valenton demeure une œuvre d'Auzelle très préservée et pourtant largement méconnue, son travail à Clamart étant systématiquement mis en avant. Si certains carrés peuvent ressembler à un cimetière ordinaire, il suffit de s'en éloigner et de se diriger vers les espaces paysagers ou le columbarium pour mesurer tout l'intérêt du projet. Quarante ans après leur plantation, les parties boisées, bien entretenues, ont atteint leur maturité. La sculpture monumentale de Székely est intacte. Les bâtiments de service, ceux de la conservation et des commerces n'ont subi aucune intervention irréversible. L'aire de cérémonie est demeurée presque inchangée jusqu'en 2014, 
conservant même en état de fonctionnement son système de cloisons mobiles. Cet espace a été restauré tout récemment; le projet prévoyait d'en conserver toutes les caractéristiques, quitte à seulement occulter visuellement certains éléments.

Ainsi, le cimetière intercommunal de Valenton mériterait davantage de reconnaissance, qui pourrait passer par une labellisation Patrimoine $d u x^{e}$ siècle, par exemple. Celle-ci aurait le mérite d'attirer l'attention du public, comme de ses gestionnaires, sur le site et d'éviter que d'éventuelles futures restructurations ne le dénaturent. Les vertus pédagogiques de cette distinction, accompagnée de la mise en place d'une signalétique didactique, faciliteraient sans doute la compréhension des intentions d'Auzelle et aideraient à la gestion et à l'entretien de la nécropole. L'effet de dépouillement propice au recueillement que l'architecte rechercha est aujourd'hui effectivement critiqué par les usagers, aspirant à des espaces moins sévères, aussi bien pour les salles de cérémonie du funérarium que pour les aires d'inhumation ${ }^{47}$. Cette nouvelle approche des intentions de l'architecte pourrait également s'étendre à la gestion des plantations qui gagneraient sans doute à bénéficier de l'expertise d'un paysagiste spécialiste des jardins patrimoniaux.

\section{NOTES}

1. - Sur l'œuvre de Robert Auzelle voir les publications du séminaire Robert Auzelle Arturbain.fr (Association pour la promotion de l'art urbain et de l'éthique du cadre de vie) et les travaux de Frédéric Bertrand, notamment: BERTRAND, Frédéric. L'architecture et l'urbanisme funéraires parisiens à l'ère des métropoles. Regard sur l'œuvre et la pensée de Robert Auzelle (1913-1983). Thèse d'urbanisme. Paris : Paris VIII, 2003. Par ailleurs, le fonds d'atelier de l'architecte est conservé au centre d'archives de la Cité de l'architecture et du patrimoine (fonds Robert Auzelle 242 IFA).

2. - AUZELLE, Robert. Les problèmes de sépulture en urbanisme. Thèse d'urbanisme. Paris : Institut d'urbanisme de l'université de Paris, 1942. Id. Dernières demeures. Conception, composition, réalisation du cimetière contemporain. Paris : Robert Auzelle, 1965.

3. - Cette étude a donné lieu à la rédaction de quatre dossiers d'Inventaire général IA94000516 et IA94000517, IM94001342 et IM94001343 qui ne sont pas encore mis en ligne sur le site de la région Île-de-France à la date de publication de cet article et qui comprendront une importante iconographie. L'auteur remercie Mme Rateau, conservatrice du cimetière, pour son accueil et les renseignements fournis.

4. - « a - Le cimetière parc. - Le Park cemetery est américain d'origine. Composé à la manière d'un jardin à l'anglaise, il permet d'implanter une riche et belle végétation qui masquera sérieusement la laideur souvent arrogante des tombeaux et des monuments funéraires. Cependant il reste une imitation trop littérale du parc anglais pour évoquer à proprement parler l'idée de cimetière telle que les Européens peuvent la concevoir. Il entre d'ailleurs dans le cadre de ces tentatives de négation de la mort, de ce matérialisme reposant qui caractérisent si fortement les réactions yankees devant les problèmes de l'outre-tombe. $\mathbf{b}$ - Le cimetière forestier. - [...] les Allemands préférèrent bientôt à cette solution [le cimetière parc] une formule de type plus germanique. L'éternel romantisme allemand $d u$ "Wald» et peut-être aussi on ne sait quelle mythologie folklorique ou universelle de l'arbre les amènent à utiliser la forêt comme décor de cimetière, enrichi d'œuvres sculpturales ou architecturales. [...] Trop de lyrisme s'y 
mêle sans doute, et trop de contingences aussi, car comment trouver à proximité d'une agglomération une forêt qui puisse être affectée à l'inhumation ? S'agit-il d'en planter une ? Pour qu'une pépinière naissante se transforme en forêt, il faut le temps d'une génération : la solution n'est donc applicable qu'à certaines parties seulement d'un cimetière nouveau dont la mise en service peut être différée pendant vingt ou trente ans. [...] c - Le cimetière architectural - Il est ainsi appelé, faute d'un meilleur terme, et il correspond à une composition basée essentiellement sur des ensembles de tombes délimités par des haies ou des arbres taillés, s'étageant à différentes hauteurs, sur de petites terrasses, un peu à la manière de jardins provençaux ou italiens. L'élément essentiel de la composition est cette fois ramené au groupe de tombes, groupe plus ou moins dispersé, où l'on demande à la nature, ici de dégager une perspective, là de masquer tel élément inégal ou défectueux. Tout cela paraît a priori assez satisfaisant mais exige un terrain constitué d'une succession de plans horizontaux ou tout au moins de faible pente dont on peut fort bien ne pas disposer. C'est pourquoi les grandes surfaces que requiert un cimetière urbain ou intercommunal entraînent presque nécessairement l'intégration dans une même composition de ces trois types de cimetière. [...] Une autre formule, non systématique ou mixte, pourrait être appelée le cimetière paysager, afin de signifier par là qu'il s'agit de dégager grâce à un accompagnement végétal un ensemble de paysage respirant une atmosphère de recueillement et de paix. D'ailleurs, à l'échelle des réalisations intercommunales, il s'agit avant tout d'une fédération de cimetières, et c'est cette solution paysagiste un peu hybride qui respecte encore le mieux le souvenir et le charme de nos anciens enclos mortuaires ceinturant les églises provinciales et qui peut en rappeler l'inspiration, le cadre de verdure et éventuellement quelques éléments architecturaux. Ce qui, transposant le passé en l'adaptant, permet de jouer non seulement avec les formes mais encore avec les volumes, les matières et les coloris. » (AUZELLE, Robert. Dernières demeures. Op. cit., p. 212-213).

5. - Ibid., p. 99.

6. - Ibid., p. 101.

7. - Sur les cimetières intercommunaux de la région parisienne voir : BERTRAND, Frédéric. Op. cit. et BELLANGER, Emmanuel. La mort, une affaire publique. Histoire du syndicat intercommunal funéraire de la région parisienne. Ivry-sur-Seine : les éditions de l'Atelier, 2008.

8. - Le conseil municipal sollicite l'adhésion de la commune au syndicat intercommunal le 11 juillet 1958. (Délibération du conseil municipal $n^{\circ} 1841 ; \mathrm{D} 1 / 32$ ).

9. - L'architecte est alors professeur assistant à l'École nationale des beaux-arts et collabore avec Auzelle pour ses travaux du séminaire de l'atelier Tony Garnier.

10. - Projet non réalisé.

11. - AUZELLE, Robert. Dernières demeures. Op. cit., chap. 6 et suiv., dont le chap. 12 : «l'une des solutions rationnelles, dictée par l'expansion démographique et la densité actuelles est la création de cimetières intercommunaux... ». Voir également BERTRAND, Frédéric. Op. cit.

12. - Auzelle accorde une place importante à l'ossuaire collectif, dispositif qu'il souhaiterait voir transformé en "mémorial nécrologique " ou "état civil de la mort » au fur et à mesure de la reprise des concessions et du transfert des restes des corps.

13. - Notamment par la société Le fibro-ciment de Poissy (Yvelines).

14. - La mauvaise desserte routière du cimetière était le premier argument "prétexte " avancé par l'administration pour rejeter le projet de Créteil. En effet l'unique route donnant accès à la nécropole aurait traversé le grand ensemble du Mont-Mesly, or elle n'avait pas été conçue pour un tel trafic. À Valenton, le choix d'un nouveau terrain contigu au nœud autoroutier levait cette difficulté.

15. - Jeune architecte qu'il encourage à suivre son séminaire de l'École des beaux-arts et qu'il recrute peu après. 
16. - La législation imposant la construction d'un haut mur de clôture depuis le XviII ${ }^{\mathrm{e}}$ siècle ne fut modifiée qu'en 1921, autorisant désormais le grillage métallique renforcé par un écran d'arbustes épineux ou à feuilles persistantes.

17. - Le second argument "prétexte" avancé par l'administration pour rejeter le projet de Créteil était le déficit de places de stationnement prévues dans le projet.

18. - Espace appelé salle omniculte dans certains projets. L'architecte rêva d'abord de cet «aireabri » polyvalent avant de pouvoir le mettre en œuvre. Il lui attribuait «tout naturellement une destination usuelle et non occasionnelle. Ce serait d'abord le lieu tout désigné d'un adieu dans le cas d'un enterrement civil. Le lieu, également, de ces rassemblements commémoratifs qui groupent, à intervalle d'anniversaires, les fidèles de tel défunt illustre, poète, artiste, homme politique. Ce pourrait être aussi, dans le cas d'un enterrement religieux, l'endroit où - si le décédé est un notable - les discours d'usage seraient prononcés. [...] Bien que les messes d'enterrement soient revendiquées assez âprement par les curés de paroisses [...] nous savons que certains prêtres pensent qu'une chapelle de cimetière disposant d'un service assuré par roulement serait, dans un grand nombre de cas de la plus nette utilité. » (AUZELLE, Robert. Dernières demeures. Op. cit., p. 301).

19. - Outre l'observation de la réalisation elle-même, ces modifications peuvent être détaillées grâce au dossier d'adjudication datant d'octobre 1971 (SIAF/CAPA. Fonds Robert Auzelle 242 IFA. Carton 61, affaire RA97).

20. - AUZELLE, Robert. Dernières demeures. Op. cit., p. 370 (chap. 21 : l'architecture funéraire tombeau - bâtiments).

21. - Auzelle, catholique non pratiquant, était aussi attaché à une certaine dimension chrétienne pour les valeurs morales et le rituel qu'elle incarnait. Sur le plan formel, le bâtiment des cérémonies est à rapprocher du courant de renouveau de l'architecture religieuse des années 1950-1960. Auzelle connaissait la chapelle Saint-Paul-d'Ambourget d'Aulnay-sous-Bois, construite en 1963-1965 par Jean Le Couteur et Paul Herbé, dont la toiture, supportée par une charpente simplissime en lamellé-collé, est formée de deux plans courbes. On peut également rapprocher son bâtiment de l'église paroissiale Sainte-Thérèse-du-Vert-Galant à Tremblay-enFrance, réalisée au tournant de 1970 par Louis-Pierre Grobois et Guy Angenendt. Ici, la charpente en lamellé-collé, au dessin beaucoup plus complexe, supporte un éclairage zénithal très similaire à celui de Valenton.

22. - Réalisés par l'entreprise parisienne Drecq (SIAF/CAPA. Fonds Robert Auzelle 242 IFA. Carton 62. Plans d'exécution datés du 9 janvier 1974).

23. - Pour leur travail et la présentation de leur fonds conservé au Centre Georges-Pompidou, bibliothèque Kandinsky, voir: GIARD, Noémie. «Photographies d'architecture. Le fonds Véra Cardot et Pierre Joly ». Études photographiques, 18 mai 2006, [En ligne], mis en ligne le 20 juin 2008. URL : http://etudesphotographiques.revues.org/2563 [consulté le 2 mars 2015].

24. - SIAF/CAPA. Fonds Robert Auzelle 242 IFA. Carton 57, affaire RA82.

25. - CEuvres aujourd'hui détruites.

26. - Pour l'œuvre du sculpteur voir notamment : Catalogue raisonné des œuvres du sculpteur Pierre Székely. http://j.p.karinthi.free.fr/ [consulté le 2 mars 2015].

27. - Ille-et-Vilaine.

28. - Ouest-France, 27 novembre 1973.

29. - L'artiste brevète en 1966 un chalumeau lui permettant de tailler le granit qu'il utilisera tout au long de sa carrière pour réaliser, parfois sur site, ses œuvres monumentales.

30. - Le mausolée. Revue mensuelle des arts et techniques des roches de qualité, juin 1975, nº 466, p. 1201-1212.

31. - Lettre d'Auzelle à Székely du 18 avril 1974 (SIAF/CAPA. Fonds Robert Auzelle 242 IFA. Carton 57, affaire RA82). 
32. - Pour l'œuvre du sculpteur voir : ORGEVAL, Domitille d'. Pierre Sabatier, sculpteur. Paris : Éd. Norma, 2011 ; http://www.pierresabatier.com/accueil.htm [consulté le 2 mars 2015].

33. - Le groupe publie la revue du même nom à partir de 1966.

34. - Cependant le clergé renâcle à utiliser ces espaces qui serviront bien davantage aux cérémonies laïques et à quelques rassemblements œcuméniques.

35. - SIAF/CAPA. Fonds Robert Auzelle 242 IFA. Carton 57, affaire RA82.

36. - SIAF/CAPA. Fonds Robert Auzelle 242 IFA. Carton 57, affaire RA79 ; carton 58, affaire RA82.

37. - Syndicat intercommunal du cimetière de Bonneuil-sur-Marne, Charenton-le-Pont, Créteil, Joinville-le-Pont, Maisons-Alfort, Saint-Maur-des-Fossés, Saint-Maurice. Cimetière intercommunal de la Fontaine-Saint-Martin situé sur les communes de Valenton, Limeil-Brévannes et Villeneuve-SaintGeorges. Réglementation des sépultures, album de modèles de tombes, architectes Robert Auzelle et Hector Patriotis.

38. - Confédération de l'artisanat et des petites entreprises du bâtiment - Section Marbre-GranitPierre. Les nouveaux cimetières, [1973].

39. - Lettre du préfet du Val-de-Marne au président du syndicat du 23 juillet 1973 (SIAF/CAPA. Fonds Robert Auzelle 242 IFA. Carton 64. Affaire RA110).

40. - Weser S.A. Les éléments de façade en béton, Mazières-de-Touraine, 37130 Langeais.

41. - Auzelle dépose plusieurs brevets à l'INPI, il interroge le conseil supérieur de l'ordre des architectes afin de connaître le meilleur moyen, sans contrevenir à la déontologie de la profession, d'être rémunéré pour son «invention » et fait réaliser des prototypes successifs. Il confie à la société Weser le soin de fabriquer et commercialiser ses inventions, contre le versement d'un pourcentage sur les ventes (SIAF/CAPA. Fonds Robert Auzelle 242 IFA. Carton 61, affaire RA96, alvéoles mortuaires préfabriquées).

42. - Interdite par l'Église catholique jusqu'en 1963 et longtemps insignifiante en France, la crémation représente aujourd'hui entre $0 \%$ des opérations funéraires en Corse et jusqu'à $32 \%$ en Alsace, sous l'influence allemande.

43. - Il dessina son propre monument funéraire familial placé dans le cimetière de Clamart au milieu d'une pelouse : trois volumes géométriques primaires encerclés par un muret bas.

44. - La parcelle initiale a été réduite au nord et la surface soustraite accueille le nouveau centre de formation et de soutien des Sapeurs-Pompiers de Paris (voir la fig. 6).

45. - L'architecte, sûr que la pratique de la crémation se développerait en France, prévoyait un crématorium dans chacun de ses projets, sans jamais pouvoir les réaliser.

46. - Sépulture cinéraire, ce petit caveau individuel construit en pleine terre accueille une ou plusieurs urnes.

47. - Les filles d'Auzelle veillent au devenir de l'œuvre de leur père. Elles sont récemment intervenues auprès de l'administration du cimetière pour souligner l'incongruité des fresques aux motifs floraux peintes sur les murs entourant l'enclave de service (abris des poubelles, point d'eau...) dans chaque carré. Ces décors contreviennent à l'esprit de sobriété voulu par l'architecte, incarné dans l'usage du béton brut.

\section{RÉSUMÉS}

L'architecte français Robert Auzelle (1913-1983) est reconnu pour son travail d'urbaniste notamment mis en œuvre à partir des années 1950 dans le quartier de la Plaine à Clamart (Hauts- 
de-Seine). Il est également réputé comme spécialiste de l'architecture funéraire grâce à ses écrits et à la réalisation, à partir de 1946, du premier véritable cimetière paysager français $d u \mathrm{xx}^{\mathrm{e}}$ siècle, toujours à Clamart. En revanche, ses autres nécropoles, telle celle de Valenton (Val-de-Marne) demeurent très peu connues. Dans cette ville du sud-est parisien, Auzelle déploie toutes ses idées pour réinventer le cimetière français, cimetière dans lequel il voudrait faire régner un esprit des lieux retrouvé, une harmonie propice au recueillement et à une forme de sacralité restaurée ; ce «cimetière paysager" proposerait la synthèse entre le «cimetière parc » américain, celui "forestier», de tradition germanique, et le «cimetière architectural» méditerranéen. L'architecte tente en outre d'y développer une approche renouvelée des sépultures elles-mêmes. Le projet, réalisé au début des années 1970, se conforme dans l'ensemble à ses dessins. Les interventions artistiques y occupent une place importante, Auzelle intégrant des sculptures monumentales commandées à des plasticiens qu'il choisit soigneusement - Pierre Székely (1923-2001) pour « Les ages de la vie » à l'entrée du cimetière et Pierre Sabatier (1925-2003) pour les paravents «l'arbre de vie » et « le cosmos » dans l'aire de cérémonie. Cependant, il échoue ici en grande partie dans sa volonté de renouveler l'art funéraire, ne réussissant pas à multiplier les tombes superposées en enfeus et à imposer - aux familles comme aux marbriers - ses dessins pour l'ensemble des sépultures. Aujourd'hui, le cimetière s'étend sur 32 hectares et dispose d'un crématorium (créé en 1986 dans l'étage semi-enterré du funérarium), d'espaces traditionnels d'inhumation (dont un israélite et un musulman), d'espaces paysagers d'inhumation, d'un ossuaire, d'un columbarium, d'enfeus et d'un site cinéraire (avec une rocaille de dispersion, des emplacements pour des cavurnes ou cavotins et un jardin des innocents). Malgré quelques modifications par rapport à son état d'origine, il demeure une œuvre d'Auzelle très préservée. Si certains carrés peuvent ressembler à un cimetière ordinaire, il suffit de s'en éloigner et de se diriger vers les espaces paysagers ou le columbarium pour mesurer tout l'intérêt du projet. Quarante ans après leur plantation, les parties boisées, bien entretenues, ont atteint leur maturité. Les bâtiments de service, ceux de la conservation et des commerces, l'aire de cérémonie n'ont subi aucune intervention irréversible. Ainsi, le cimetière intercommunal de Valenton n'attend plus qu'une meilleure reconnaissance, qui pourrait passer par une labellisation Patrimoine $d u x^{e}$ siècle, par exemple. Les vertus pédagogiques de cette distinction faciliteraient sans doute la compréhension des intentions d'Auzelle et aideraient à la gestion et à l'entretien de la nécropole. L'effet de dépouillement propice au recueillement que l'architecte rechercha est aujourd'hui effectivement critiqué par certains usagers, aspirant à des espaces moins sévères.

The French architect Robert Auzelle (1913-1983) is well known for his works as town planner, implemented particularly in the fifties, in the district of "La Plaine" in Clamart (Hauts-de-Seine). $\mathrm{He}$ is also renowned as a specialist in funerary architecture thanks to his papers and to his realization of the first real French landscaped cemetery of the XXth century, begun in 1946, still in Clamart. However his other necropolises, such as the one of Valenton (Val-de-Marne) remain little known. In this city located in the southwestern suburb of Paris, Auzelle develops his ideas to reinvent French cemeteries, where he wants to create a place of reunion, a location suitable for recollection and a renovated expression of the sacred character. This "landscaped cemetery" would propose the synthesis between the American "park cemetery", the "forest one" of Germanic tradition, and the "architectural cemetery" typical of Mediterranean countries. In addition, the architect tries to develop a renewed approach to graves themselves here. The project, realized at the beginning of the seventies, conforms altogether to his drawings. The artistic interventions play an important role here, with Auzelle integrating monumental sculptures ordered to visual artists whom he chooses carefully: Pierre Székely (1923-2001) for "The Ages of Life" at the entrance of the cemetery and Pierre Sabatier (1925-2003) for the folding screens "The Tree of Life" and "The Cosmos" in the space of ceremony. However, here he largely 
fails in his will to renew the funeral art, not managing to multiply the wall-niche tombs and to impose - on families as well as on marble masons - his drawings for all the graves. Today the cemetery stretches out on over 32 hectares and presents: a crematorium (created in 1986 in the half-buried floor of the funeral parlor), traditional spaces of burial (among which a Jewish and a Muslim one), landscaped spaces of burial, an ossuary, a columbarium, wall-niche tombs and a funerary site (with a rocky ground for ashes scattering and sites for volts or urns and a children's burial grounds). In spite of some modifications compared to its original state, it remains a very protected Auzelle's work. Even if certain funeral plots can be like an ordinary cemetery, one only needs to move away from them and head for the landscaped spaces or the columbarium to understand all the interest of the project. Forty years after planting, the wooded parts, well maintained, have reached their maturity. The service buildings, those in charge of preservation and business and the area of ceremony have not undergone any irreversible intervention. Therefore, the inter-communal cemetery of Valenton only awaits a better recognition, which could come from the Heritage of the XXth century label, for example. The educational virtues of this distinction would facilitate, without any doubt, the understanding of Auzelle's intentions and would help the management and the maintenance of the necropolis. The effect of sobriety favorable to reverence, which the architect looked for, today is actually criticized by certain users, aspiring to less severe spaces.

\section{INDEX}

Keywords : landscape cemetery, Auzelle Robert (architect), Székely Pierre (sculptor), Sabatier Pierre (sculptor), funeral home, mortuary, Valenton (Val-de-Marne), XXe century, columbarium, grave

Mots-clés : cimetière paysager, Auzelle Robert (architecte), Székely Pierre (sculpteur), Sabatier Pierre (sculpteur), funérarium, Valenton (Val-de-Marne), XXe siècle, colombarium, tombeau

\section{AUTEUR}

\section{ISABELLE DUHAU}

Chargée de mission, Mission Inventaire général du patrimoine culturel, Service du Patrimoine, Direction générale des Patrimoines isabelle.duhau@culture.gouv.fr 